\title{
Identification of aerodynamic damping in wind turbines using time-frequency analysis
}

\author{
Bei Chen ${ }^{\mathrm{a}, *}$, Zili Zhang ${ }^{\mathrm{b}}$, Xugang Hua ${ }^{\mathrm{a}}$, Biswajit Basu ${ }^{\mathrm{c}}$, Søren R.K. Nielsen ${ }^{\mathrm{d}}$ \\ ${ }^{a}$ Key Laboratory for Wind and Bridge Engineering, Hunan University, 410082 Changsha, China \\ ${ }^{b}$ Department of Engineering, Aarhus University, 8000 Aarhus, Denmark \\ ${ }^{c}$ Department of Civil, Structural and Environmental Engineering, School of Engineering, Trinity College Dublin, \\ Dublin 2, Ireland \\ ${ }^{d}$ Department of Civil Engineering, Aalborg University, 9000 Aalborg, Denmark
}

\begin{abstract}
The paper presents a wavelet-based linearization method for evaluating aerodynamic damping of a wind turbine during operation. The method is used to estimate the aerodynamic damping solely from actual measurements of the dynamic response of the operating wind turbine due to ambient excitation from air turbulence and control forces. Based on the response measurements the generalised displacement, velocity and acceleration vectors related to a given aeroelastic model and an available aeroelastic code are estimated by a state observer. Then, the external generalised load vector, depending on the generalised velocity vector, is obtained from the aeroelastic code. Next, the external generalised load vector is linearized into two parts: a quasi-static load vector independent on the generalised velocity vector and a first order term linearly proportional to the velocity vector indicating the aerodynamic damping matrix. Filtering technique is applied to evaluate the quasi-static load vector from the actual measurements of the structural stiffness force, made up as a product of the time-dependent stiffness matrix and the estimated generalised displacement vector. Finally, the time-dependent aerodynamic damping matrix has been evaluated by wavelet analysis at each time step. Unlike other inverse-based approaches, this wavelet-based method can avoid calculating the inverse of the velocity vector covariance matrix, which is singular. The proposed method has been illustrated by a reduced 13-DOF aeroelastic model, which is used to mimic the in-situ response measured on the wind turbine.
\end{abstract}

Keywords: aerodynamic damping, system identification, wavelet analysis, least square, wind turbines

\section{Introduction}

Damping is the most uncertain parameter in a numerical model of a structure. It plays an important part in most dynamically-sensitive civil and mechanical structures. Characterization of damping in wind turbines is particularly complicated as the aerodynamic damping may vary with

\footnotetext{
Corresponding author, Tel:+86 15116313452

Email addresses: chamber@hnu.edu.cn (Bei Chen), zili_zhang@eng.au.dk (Zili Zhang), cexghua@hotmail.com (Xugang Hua), basub@tcd.ie (Biswajit Basu), srkn@civil.aau.dk (Søren R.K. Nielsen) 
time and can even be negative depending on the aeroelastic response. The negative aerodynamic damping may lead to stall-induced vibrations in turbines, which are single mode instability in the flapwise or edgewise displacement components [1,2]. Experimental and numerical simulations have shown that these vibrations are also related to certain vibration modes of the turbine. The flapwise vibrations of the blade modes are often more damped than the edgewise vibrations $[1,3]$. Both the aerodynamic characteristics of the blade profiles and the structural dynamics of the blades influence the aerodynamic damping in wind turbines[4].

Evaluation of aerodynamic damping has primarily been based on a numerical analysis in connection with a given aeroelastic model. Based on the linear aeroelastic model, Hansen [5] carried out the Multi-blade coordinate transformation (MBC) (also known as the Coleman transformation [6]) to obtain a time-invariant representation of wind turbine system, from which the aerodynamic damping ratios were determined. Kühn [7] extended the work to consider the full non-linear property of the system. Specifically, the full non-linear system was forced into resonance at a specific eigen-frequency. Next, free-decay vibration signal was analysed to evaluate the logarithmic decrement, whereby the damping ratio can be estimated accordingly. Tempel and Salzmann $[8,9]$ presented some more straightforward methods. They presented a formulation for the aerodynamic damping ratios for the traditional constant speed turbines and applied some aeroelastic codes to determine the aerodynamic damping value.

Alternatively, the damping may be obtained from experimental data from the operating wind turbine. Studies by James et al. [10] provided a practical approach to extract damping ratios by treating the auto- and cross-covariance function as free vibration responses. Based on set of strain-gauge signals, they calculated the auto- and cross-spectral density function of the obtained response realization. Next, the related auto- and cross-covariance functions were obtained by Fourier transformation. Finally, the poly reference time domain method [11], a kind of system identification technique based on the impulse response functions due to multiple exciter locations is applied to extract the damping ratios (both structure and aerodynamic) from the autocovariance and cross-covariance functions which are treated as free vibration responses. Hansen et al. [12] proposed two experimental methods for estimating the aeroelastic damping. The first method is based on using two types of excitation to excite the turbine during operation at the natural frequency of a specific mode. Next, the excitation is stopped and decaying vibrations at eigen frequency are measured, whereby the damping can be estimated. The second method based on the Stochastic Subspace Identification (SSI) analysis is used to extract the natural frequencies and damping of the turbine modes solely from the response of the operating turbine due to the ambient excitation from air turbulence. In the study by Thomsen et al. [13], three different types of excitation were used to excite the edgewise vibration of the blade at a specific mode and stop the exciter in order to get the free-decaying signal, which contains the total damping (i.e. structure damping and aerodynamic damping) of the vibration mode. By using an exponential function to fit the decay amplitudes, they obtained the damping ratios of the considered mode.

Recently, wavelet analysis is increasingly being employed by engineers in the field of system identification, and a number of studies have been made for extracting the damping property [14-17] because of its time-frequency analysis capability and the ability to extract features from non-stationary data. Ruzzene et al. [14] proposed an approach to estimate the equivalent viscous damping ratios by taking wavelet transformation of the impulse response obtained from a viscously damped single degree of freedom system with the Morlet wavelet. The transformation 
results in an exponential function, which contains the damping ratio. Therefore, damping ratio can be estimated by calculating the slope of the exponential function in a logarithmic scale plot. Chakraborty et al. [15] provided a similar study where the estimation of modal damping and modal shape was carried out by using a modified L-P mother wavelet. However, most works are focused on tall buildings, transmission towers and long-span bridges [18-21]. Limited studies have been carried out in connection with the wind turbines, especially the aerodynamic damping identification. A major contribution in this field is the work of Murtagh and Basu [22], where two damping identification approaches are introduced: one which is Fourier based and the other wavelet based, primarily applicable to a multi-DOF linear structure with closely spaced modes of vibration. The first method is based on the Fourier transformation of the motion equation, by using least square method to estimate the equivalent viscous damping ratios. The second method is based on the wavelet method, using a time-segmented least square approach to extract the damping ratios.

This paper proposes a methodology for identification of the time-variant aerodynamic damping matrixes of a wind turbine using the wavelet-based equivalent linearization technique. The method presumes that the structural property matrix including the mass matrix, structural damping matrix and stiffness matrix have been obtained from a given aeroelastic code. Based on in-situ measurements of the dynamic response, the external load is linearized in the generalised velocity vector to separate the aerodynamic damping force from the non-linear generalised external load vector. Finally, the aerodynamic damping matrix is identified from the aerodynamic damping force vector by a wavelet transform technique. The proposed estimation approach is particularly useful to wind turbines when the aerodynamic damping may vary with time depending on the operational conditions.

\section{The 13-DOF wind turbine model}

\subsection{General description}

The wind turbine system is modelled as a 13-DOF aeroelastic model as shown in Fig. 1. The motions of the tower and the drive train are described in a fixed, global $\left(X_{1}, X_{2}, X_{3}\right)$-coordinate, while the elastic motion of each blade relative to the hub is indicated in a moving, local $\left(x_{1}, x_{2}, x_{3}\right)$ coordinate system with its origin at the hub. The local $x_{1}$-axis is co-directional to the global $X_{1}$ axis, and the $x_{3}$-axis is orientated from the hub towards the tip of the blade. Assuming a constant nominal rotational speed $\Omega$ of the rotor, the position of the local coordinate system attached to blade $j$ is specified by the azimuthal angle $\Psi_{\mathrm{j}}(t)[23]$ :

$$
\Psi_{\mathrm{j}}(t)=\Omega t+\frac{2 \pi}{3}(j-1), j=1,2,3
$$

which is positive when rotating clockwise as observed from an upwind position. In reality $\Psi_{\mathrm{j}}(t)$ contains a contribution from the elastic rotation of the rotor shaft at the hub, which is assumed to be negligible.

Each blade is modelled as a Bernoulli-Euler beam with variable mass per unit length and variable bending stiffness. The flapwise and edgewise motions of the three blades are modelled by the DOFs $q_{\mathrm{j}}(t)$ and $q_{\mathrm{j}+3}(t), j=1,2,3$, indicating the tip displacement in the positive $x_{1}$-direction 


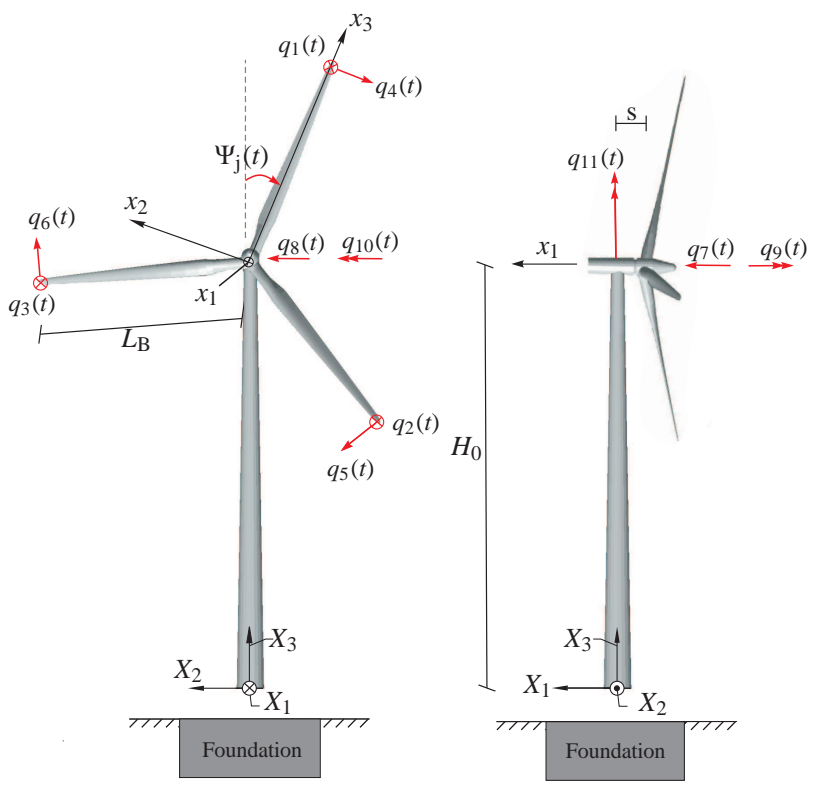

Figure 1: the 13-DOF aeroelastic wind turbine model. Definition of fixed and moving coordinate systems and the degrees of freedom $q_{1}(t), \ldots, q_{11}(t)$.

and the negative $x_{2}$-direction, respectively. The related mode shapes are taken as the undamped fixed bay fundamental eigenmodes $\Phi_{\mathrm{f}}\left(x_{3}\right)$ and $\Phi_{\mathrm{e}}\left(x_{3}\right)$ in the flapwise and edgewise directions.

The tower motion is defined by the translational DOFs $q_{7}(t)$ and $q_{8}(t)$ in the global $X_{1}$ - and $X_{2}$ directions, and the rotational DOFs $q_{9}(t), q_{10}(t), q_{11}(t)$ in the global $X_{1^{-}}, X_{2}$ - and $X_{3}$-directions. Hence, the lateral tower vibration is modelled by the top elastic displacement $q_{8}(t)$ and top elastic rotation $q_{9}(t)$, using cubic shape function [23].

The drivetrain shown in Fig. 2 is modelled by the DOFs $q_{12}(t)$ and $q_{13}(t)$, indicating the deviations of the rotational angles at the hub and the generator from the nominal rotational angles $\Omega t$ and $N \Omega t$, respectively, where $N$ is the gear ratio. Correspondingly, $\dot{q}_{12}(t)$ and $\dot{q}_{13}(t)$ are the deviations of the rotational speeds at the hub and the generator from the nominal values. $J_{\mathrm{r}}$ and $J_{\mathrm{g}}$ denote the mass moment of inertia of the rotor and the generator, and $k_{\mathrm{r}}$ and $k_{\mathrm{g}}$ denote the St.Venant torsional stiffness of the rotor shaft and the generator shaft.

Assuming linear structural dynamics and substituting the kinetic and potential energies into the Euler-Lagrange equation, the equations of motion of the 13-DOF model are obtained by the form [23]:

$$
\mathbf{M}(t) \ddot{\mathbf{q}}(t)+\mathbf{C}(t) \dot{\mathbf{q}}(t)+\mathbf{K}(t) \mathbf{q}(t)=\mathbf{F}(t, \dot{\mathbf{q}}(t))
$$

where $\mathbf{q}(t)$ is the column vector of the degree of freedom. $\mathbf{M}(t)$ is the mass matrix, $\mathbf{C}(t)$ is the damping matrix including the structural and gyroscopic damping, and $\mathbf{K}(t)$ is the stiffness matrix taking into account the geometric and gyroscopic stiffness. $\mathbf{F}(t, \dot{\mathbf{q}}(t))$ is the external generalised 


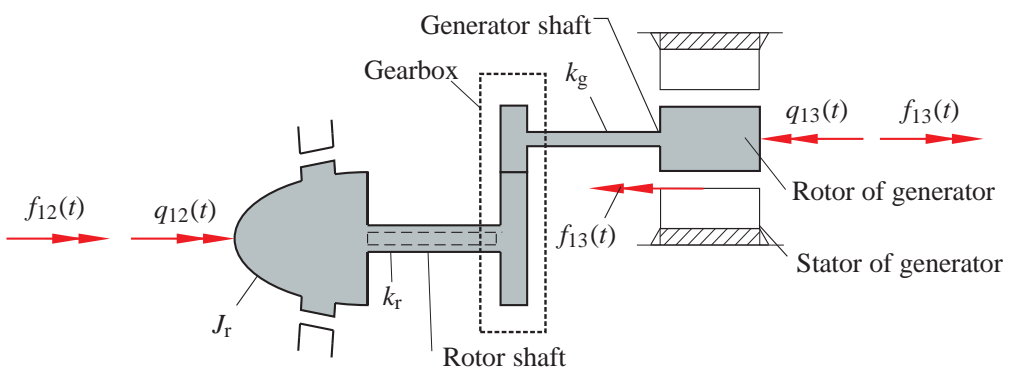

Figure 2: 2-DOF model of the flexible drivetrain with odd number of gear stages. Definition of degrees of freedom $q_{12}(t)$ and $q_{13}(t)$.

load vector work conjugated to $\dot{\mathbf{q}}(t)$, including the non-linear aerodynamic loads and the generator torque.

All the indicated system matrices contain the azimuthal angle $\Psi_{\mathrm{j}}(t)$ and are thus time-varying. This is because the displacements of the blades are modelled in the moving coordinate system, while others are formulated in a fixed coordinate system. Detailed expressions of the system matrices can be found in [23]. The aeroelastic model as well as the developed code has been extensively benchmarked against the NREL FAST code [24], with an error margin below 5\% on the displacement components in all numerical experiments [23].

\subsection{Wind field representation}

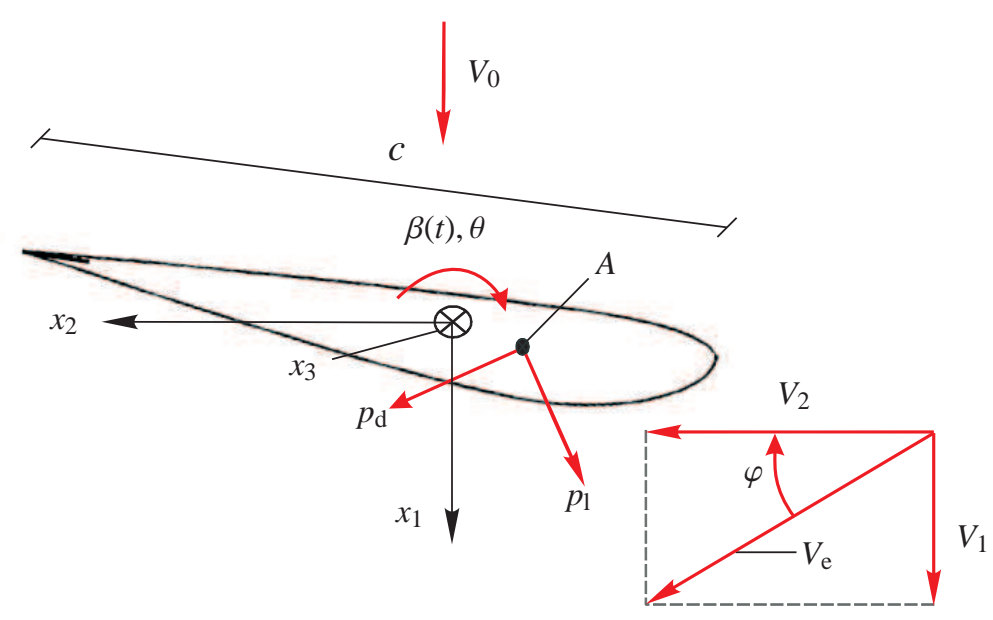

Figure 3: Wind velocities in rotor plane.

Fig. 3 shows the wind velocities in the rotor plane. $V_{\mathrm{e}}\left(x_{3}, t\right)$ denotes the resulting wind velocity on the profile. $V_{1}\left(x_{3}, t\right)$ and $V_{2}\left(x_{3}, t\right)$ are the instantaneous wind velocities in local $x_{1}$ direction and $x_{2}$-direction, respectively, seen by an observer fixed to the moving $\left(x_{1}, x_{2}, x_{3}\right)$ coordinate system. These are given as: 


$$
\left.\begin{array}{c}
V_{\mathrm{e}}\left(x_{3}, t\right)=\sqrt{V_{1}^{2}\left(x_{3}, t\right)+V_{2}^{2}\left(x_{3}, t\right)} \\
V_{1}\left(x_{3}, t\right)=V_{1,0}+v_{1}\left(x_{3}, t\right)-\dot{u}_{1}\left(x_{3}, t\right), V_{1,0}=(1-a) V_{0} \\
V_{2}\left(x_{3}, t\right)=V_{2,0}+v_{2}\left(x_{3}, t\right)-\dot{u}_{2}\left(x_{3}, t\right), V_{2,0}=\left(1+a^{\prime}\right) \Omega x_{3}
\end{array}\right\}
$$

where $V_{1,0}$ and $V_{2,0}$ indicate the mean axial wind and rotational velocity components co-directional to the moving $x_{1}$ - and $x_{2}$-axes, and $a$ and $a^{\prime}$ are the related axial and tangential induction factors [25]. $v_{\mathrm{j}}\left(x_{3}, t\right)$ indicates the components in the moving frame of reference of the rotational sampled turbulence at the position $x_{3}$, at the time $t . \dot{u}_{\mathrm{j}}\left(x_{3}, t\right)$ denotes the corresponding moving frame components of the velocity vector of the blade at the abscissa $x_{3}$, due to elastic deformations of the tower and the blade. This term is causing the aerodynamic damping. The rigid body velocity due to the rotation of the blade is included in $V_{2,0}\left(x_{3}\right)$.

The turbulence modelling is based on Taylor's hypothesis of frozen turbulence [26], corresponding to a frozen turbulence field which is convected into the rotor in the global $X_{1}$-direction with a mean velocity $V_{0}$. Additionally, the mean velocity $V_{0}$ is assumed to be constant over the rotor plane. Further, the frozen field is assumed to be a zero mean homogeneous and isotropic stochastic, with a covariance structure as presented in [27]. In accordance with this covariance structure, the 1st order AR model [28] performs a 1st order filtering of the white noise input, from which the continuous, non-differentiable sample curves of the turbulence field at the rotor plane can be generated accordingly. Next, the turbulence encountered in the moving frame of reference fixed to the rotating blade is obtained by linear interpolation between the turbulence at different grid points in the fixed frame of reference, resulting in the rotational sampled turbulence [29].

With the generated rotational sampled turbulence result, the aerodynamic loads along the blade can be calculated by the widely-used Blade Element Momentum (BEM) method with Prandtl's tip loss factor and Glauert correction [30]. This model also considered the non-linear aeroelasticity by introducing the local deformation velocities $\dot{u}_{\mathrm{j}}\left(x_{3}, t\right)$ of the blade into calculations of the flow angle and the angle of attack. As a result, this wind turbine model possesses high aerodynamic damping in the blade flapwise and the fore-aft tower vibrations, but relatively low aerodynamic damping in the blade edgewise and the lateral tower vibrations.

$p_{1}\left(x_{3}, t\right)$ and $p_{\mathrm{d}}\left(x_{3}, t\right)$ denote the instantaneous lift and drag force per unit of length on the profile. Then, these loads are calculated by means of so-called quasi-static aerodynamics as:

$$
\left.\begin{array}{l}
p_{1}\left(x_{3}, t\right)=\frac{1}{2} \rho V_{\mathrm{e}}^{2}\left(x_{3}, t\right) c\left(x_{3}\right) c_{1}(\alpha) \\
p_{\mathrm{d}}\left(x_{3}, t\right)=\frac{1}{2} \rho V_{\mathrm{e}}^{2}\left(x_{3}, t\right) c\left(x_{3}\right) c_{\mathrm{d}}(\alpha)
\end{array}\right\}
$$

where $\alpha=\alpha\left(x_{3}, t\right)$ denotes the instantaneous angle of attack, given as:

$$
\begin{gathered}
\alpha\left(x_{3}, t\right)=\varphi\left(x_{3}, t\right)-\beta(t)-\theta\left(x_{3}\right) \\
\varphi\left(x_{3}, t\right)=\underset{6}{\arctan } \frac{V_{1}\left(x_{3}, t\right)}{V_{2}\left(x_{3}, t\right)}
\end{gathered}
$$


$\varphi\left(x_{3}, t\right)$ is the flow angle, $\beta(t)$ is the pitch angle and $\theta\left(x_{3}\right)$ is the pre-twist of the blade. $\rho$ is the mass density of air and $c\left(x_{3}\right)$ is the chord length of the profile at the coordinate $x_{3} . c_{1}(\alpha)$ and $c_{\mathrm{d}}(\alpha)$ denotes the lift and drag coefficient obtained from static $2 D$ wind tunnel test data.

$\mathbf{F}(t, \dot{\mathbf{q}}(t))$ is the external generalised load vector due to the distributed loads in Eq. (5). $\dot{\mathbf{q}}(t)$ is the generalised velocity vector obtained from the discretization of the velocity field $\dot{u}_{\mathrm{j}}\left(x_{3}, t\right)$. $\mathbf{F}(t, \dot{\mathbf{q}}(t))$ becomes a stochastic vector process partly because of the dependence of the turbulence process, and partly due to the dependence on the stochastic stochastic velocity vector $\dot{\mathbf{q}}(t)$.

\subsection{Linearization of load vector}

Based on the definition of aerodynamic damping in [31] Appendix A, the external generalised load factor on the right hand side of Eq. (2) shall be linearized as follows:

$$
\mathbf{F}(t, \dot{\mathbf{q}}(t))=\mathbf{F}_{0}(t)-\mathbf{C}_{\mathrm{a}}(t) \dot{\mathbf{q}}(t)+\boldsymbol{\varepsilon}(t)
$$

$\mathbf{F}_{0}(t)$ indicates the stochastic load vector on the wind turbine, when the structure is considered infinite rigid against elastic deformations corresponding to $\dot{\mathbf{q}}(t) \equiv 0 . \mathbf{C}_{\mathrm{a}}(t)$ indicates the linear aerodynamic damping matrix and $\boldsymbol{\varepsilon}(t)$ is the remainder in the Taylor expansion. As follows from the load model in Eqs. (5),(6) and (7), $\mathbf{C}_{\mathrm{a}}(t)$ becomes time-varying due to the time-dependent pitch angle $\beta(t)$. To get the long-term properties of the aerodynamic damping this variation will be smoothed out by averaging over $1-3$ rotational periods.

The aerodynamic damping matrix $\mathbf{C}_{\mathrm{a}}(t)$ cannot be calculated directly from the Eq. (8), because of the matrix $\dot{\mathbf{q}}(t) \dot{\mathbf{q}}^{T}(t)$ is singular. Thus, section 3.1 will show that $\mathbf{C}_{\mathrm{a}}(t)$ can be effectively estimated by wavelet transforms on condition that $\mathbf{F}(t, \dot{\mathbf{q}}(t))$ and $\mathbf{F}_{0}(t)$ are known. Section 3.2 demonstrates how $\mathbf{F}(t, \dot{\mathbf{q}}(t))$ can be obtained based on actual measurements of $\mathbf{q}(t), \dot{\mathbf{q}}(t)$ and $\ddot{\mathbf{q}}(t)$. Similarly, section 3.2 that $\mathbf{F}_{0}(t)$ can be estimated from $\mathbf{F}_{\mathrm{s}}(t, \mathbf{q}(t))=\mathbf{K}(t) \mathbf{q}(t)$, if the dynamic response component in this response vector is filtered out.

\section{Evaluation of aerodynamic damping by wavelet analysis}

\subsection{Evaluation of aerodynamic damping by using wavelet linearization}

In wavelet analysis, a signal $f(t)$, a function of time, is expressed as a composition of several time localized shifted and scaled basis functions, $\Psi\left(\frac{t-b}{a}\right)$ where ' $b$ ' and ' $a$ ' are the shifting and scaling parameters, respectively. Wavelet transform converts an initial data sequence representing a chosen length of input signal $f(t)$ into a new $2 D$ sequence, which consists of the wavelet coefficients $W_{\Psi} f(a, b)$ and is defined as [32]:

$$
W_{\Psi} f(a, b)=\frac{1}{\sqrt{a}} \int_{-\infty}^{+\infty} f(t) \Psi\left(\frac{t-b}{a}\right) d t
$$

The wavelet transform is an integral transform which convolutes the wavelet basis function $\Psi(t)$, called the mother wavelet, with the function $f(t)$ being analyzed to generate the wavelet coefficients. In this study, the "Mexican hat" has been chosen as mother wavelet [33]. In order to numerically compute the wavelet transform, it is necessary to discretize $a$ and $b$ which 
leads to the discretized version of the continuous wavelet transform (CWT) known as a discrete parameter wavelet transform (DPWT). In the DPWT, the scaling factor $a$ may be discretized logarithmically, as given by [34]:

$$
a_{\mathrm{m}}=\sigma^{m}
$$

where $\sigma$ is the sampling constant and $m$ is an integer. The shifting factor $b$ is also discretized as:

$$
b_{\mathrm{i}}=i \triangle b
$$

where $\Delta b$ is also a sampling time constant and $i$ is an integer. The values of the discretization parameters are taken as $\Delta b=0.02$ and $\sigma=2$ (as commonly used for the dyadic wavelets). On substituting Eq. (10) and Eq. (11) into Eq. (9) yields the expression for the DPWT, as:

$$
W_{i, m} f=\sigma^{-m / 2} \int_{-\infty}^{+\infty} f(t) \Psi\left(\sigma^{-m} t-\sigma^{-m} i \Delta b\right) d t
$$

Assuming that aerodynamic damping matrix $\mathbf{C}_{\mathrm{a}}(t)$ is deterministic at the instant, $t=b_{\mathrm{i}}$. On taking a wavelet transform of Eq. (8) and on discretizing with respect to $a$ and $b$, we obtain:

$$
W_{i, m} \mathbf{F}=W_{i, m} \mathbf{F}_{0}-\mathbf{C}_{\mathrm{a}}\left(b_{\mathrm{i}}\right) W_{i, m} \dot{\mathbf{q}}+W_{i, m} \boldsymbol{\varepsilon}
$$

The magnitude of the error term becomes:

$$
W_{i, m}^{T} \boldsymbol{\varepsilon} W_{i, m} \boldsymbol{\varepsilon}=\left(W_{i, m} \mathbf{F}-W_{i, m} \mathbf{F}_{0}+\mathbf{C}_{\mathrm{a}}\left(b_{\mathrm{i}}\right) W_{i, m} \dot{\mathbf{q}}\right)^{T}\left(W_{i, m} \mathbf{F}-W_{i, m} \mathbf{F}_{0}+\mathbf{C}_{\mathrm{a}}\left(b_{\mathrm{i}}\right) W_{i, m} \dot{\mathbf{q}}\right)
$$

It may be noted that $W_{i, m} \boldsymbol{\varepsilon}^{T} W_{i, m} \boldsymbol{\varepsilon}$, when summed over all $m$ values with a multiplying factor, $1 / a_{\mathrm{m}}$, represents the error in the instantaneous energy of the response at the instant of time, $t=b_{\mathrm{i}}$ [33]. $\mathbf{C}_{\mathrm{a}}(t)$ at the time step $t=b_{\mathrm{i}}$ is obtained as the solution to the minimization problem:

$$
\min _{\mathbf{C}_{\mathrm{a}}\left(b_{\mathrm{i}}\right)} \frac{1}{k+1} \sum_{j=i}^{i+k} \sum_{m=1}^{p} \frac{1}{a_{\mathrm{m}}} W_{i, m}^{T} \boldsymbol{\varepsilon} W_{i, m} \boldsymbol{\varepsilon}
$$

where $p$ denotes the number of wavelet bands.

By using the least square approach [33] and a smoothing technique, the solution for the aerodynamic damping matrix $\mathbf{C}_{\mathrm{a}}\left(b_{\mathrm{i}}\right)$, is given by:

$$
\mathbf{C}_{\mathrm{a}}\left(b_{i}\right)=\mathbf{W}_{\triangle \mathbf{F} \dot{\mathbf{q}}}\left(b_{i}\right) \mathbf{W}_{\dot{\mathbf{q}} \dot{\mathbf{q}}}^{-1}\left(b_{i}\right)
$$

where the subscript load vector $\triangle \mathbf{F}$ in Eq. (16) is defined as:

$$
\triangle \mathbf{F}(t, \dot{\mathbf{q}}(t))=\mathbf{F}(t, \dot{\mathbf{q}}(t))-\mathbf{F}_{0}(t)
$$

$\mathbf{W}_{\triangle \mathbf{F} \dot{\mathbf{q}}}$ and $\mathbf{W}_{\dot{\mathbf{q}} \dot{\mathbf{q}}}$ are matrices with components $W_{\triangle \mathbf{F} \dot{q}, l n}$ and $W_{\dot{\mathbf{q}} \dot{\mathbf{q}}, l n}$, given as:

$$
W_{\triangle \dot{\mathbf{F}} \dot{\mathbf{q}}, l n}\left(b_{i}\right)=-\frac{1}{k+1} \sum_{j=i}^{i+k} \sum_{m=1}^{p} \frac{1}{a_{m}} W_{j, m} \Delta F_{l} W_{j, m} \dot{q}_{\mathrm{n}}
$$




$$
W_{\dot{\mathbf{q}} \mathbf{q}, l n}\left(b_{\mathrm{i}}\right)=\frac{1}{k+1} \sum_{j=i}^{i+k} \sum_{m=1}^{p} \frac{1}{a_{\mathrm{m}}} W_{j, m} \dot{q}_{l} W_{j, m} \dot{q}_{\mathrm{n}}
$$

where $k$ denotes the total number of time intervals $\Delta b$ in the smoothing interval $T_{\mathrm{s}}$ :

$$
k=\left[\frac{T_{\mathrm{s}}}{\triangle b}\right]
$$

where [.] indicates the integer part as mentioned in Eq. (20). In the following results $T_{\mathrm{s}}=3 T$, $T$ denotes the rotational period $T=2 \pi / \Omega$ and $\Omega$ is the rated nominal rotational speed of the rotor.

Theoretically, the aerodynamic damping matrix $\mathbf{C}_{\mathrm{a}}\left(b_{i}\right)$ calculated from Eq. (16) is not the instantaneous aerodynamic damping matrix. In fact, in time-frequency analysis complete localization in time is not possible if localization in frequency is also desired. Hence, in reality $\mathbf{C}_{\mathrm{a}}\left(b_{i}\right)$ represents the time varying aerodynamic damping computed over a window in some averaged sense. In Eq. (13), contribution of $\mathbf{C}_{\mathrm{a}}(t)$ at $t=b_{i}$ is assumed to be significant, due to the decay and cancellation of the wavelet further away from $t=b_{i}$, hence $\mathbf{C}_{\mathrm{a}}(t)$ at $t=b_{i}$ is approximated by $\mathbf{C}_{\mathrm{a}}\left(b_{i}\right)$.

Since $\mathbf{C}_{\mathrm{a}}\left(b_{i}\right)$ represents the time varying aerodynamic damping computed over a time window in an "average" sense, the sum over " $j$ " in Eq. (15), Eq. (18) and Eq. (19) employs a windowed time "averaging" scheme. This is updated at every time step over the previous window of $k \cdot \Delta b$, using a sliding window.

Let $\hat{\mathbf{F}}(t, \dot{\mathbf{q}}(t))$ denote the linearized load vector of the external generalised load vector $\mathbf{F}(t, \dot{\mathbf{q}}(t))$, where the error term is ignored:

$$
\hat{\mathbf{F}}(t, \dot{\mathbf{q}}(t))=\mathbf{F}_{0}(t)-\mathbf{C}_{\mathrm{a}}(t) \dot{\mathbf{q}}(t)
$$



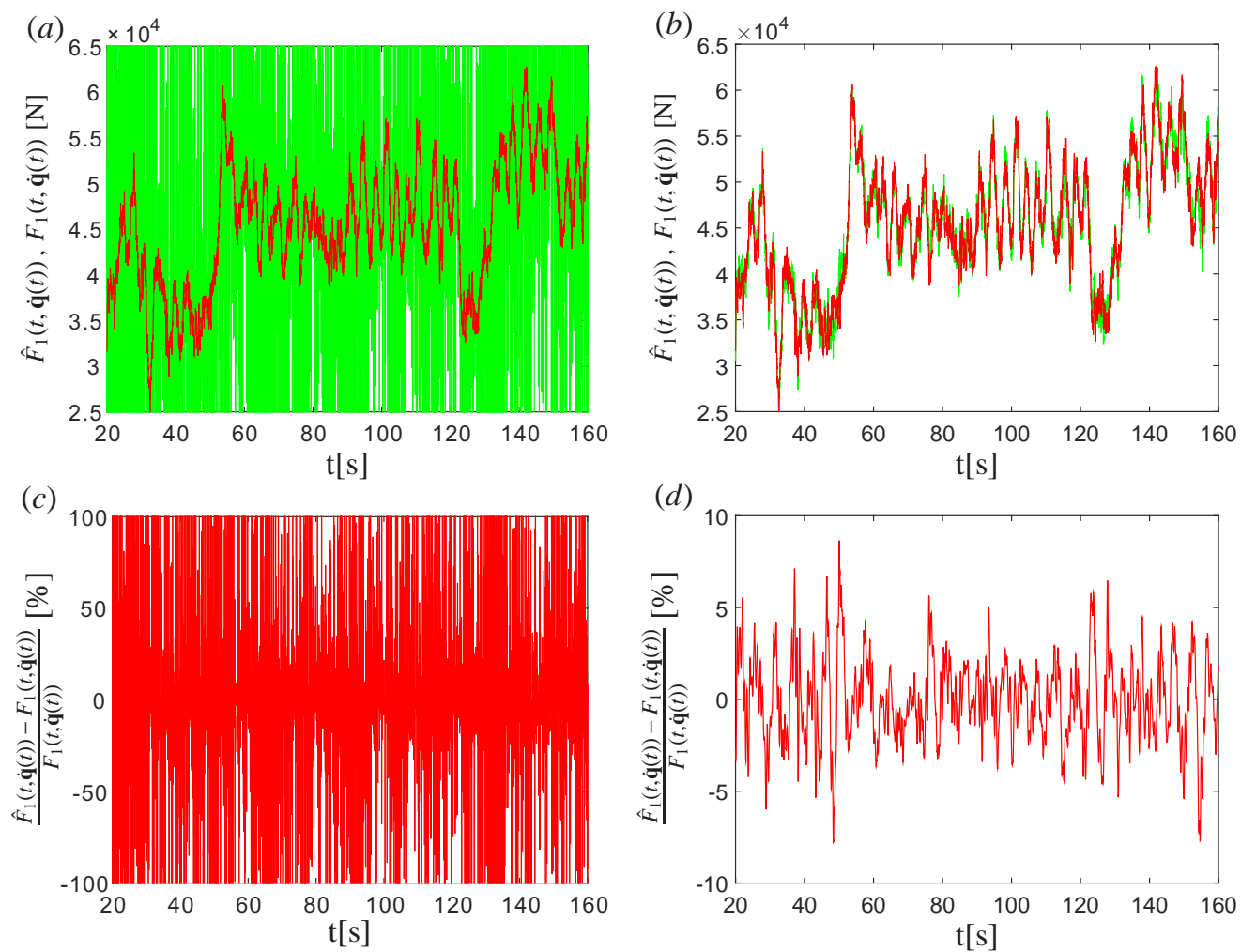

Figure 4: Flapwise load components $\hat{F}_{1}(t, \dot{\mathbf{q}}(t))$ (green), $F_{1}(t, \dot{\mathbf{q}}(t))$ (red) and load relative difference $\frac{\hat{F}_{1}(t, \dot{\mathbf{q}}(t))-F_{1}(t, \dot{\mathbf{q}}(t))}{F_{1}(t, \dot{\mathbf{q}}(t))}$ (red). (a)(c) Wavelet band value $p=5$, (b)(d) Wavelet band value $p=20$. $\xi=0$. 

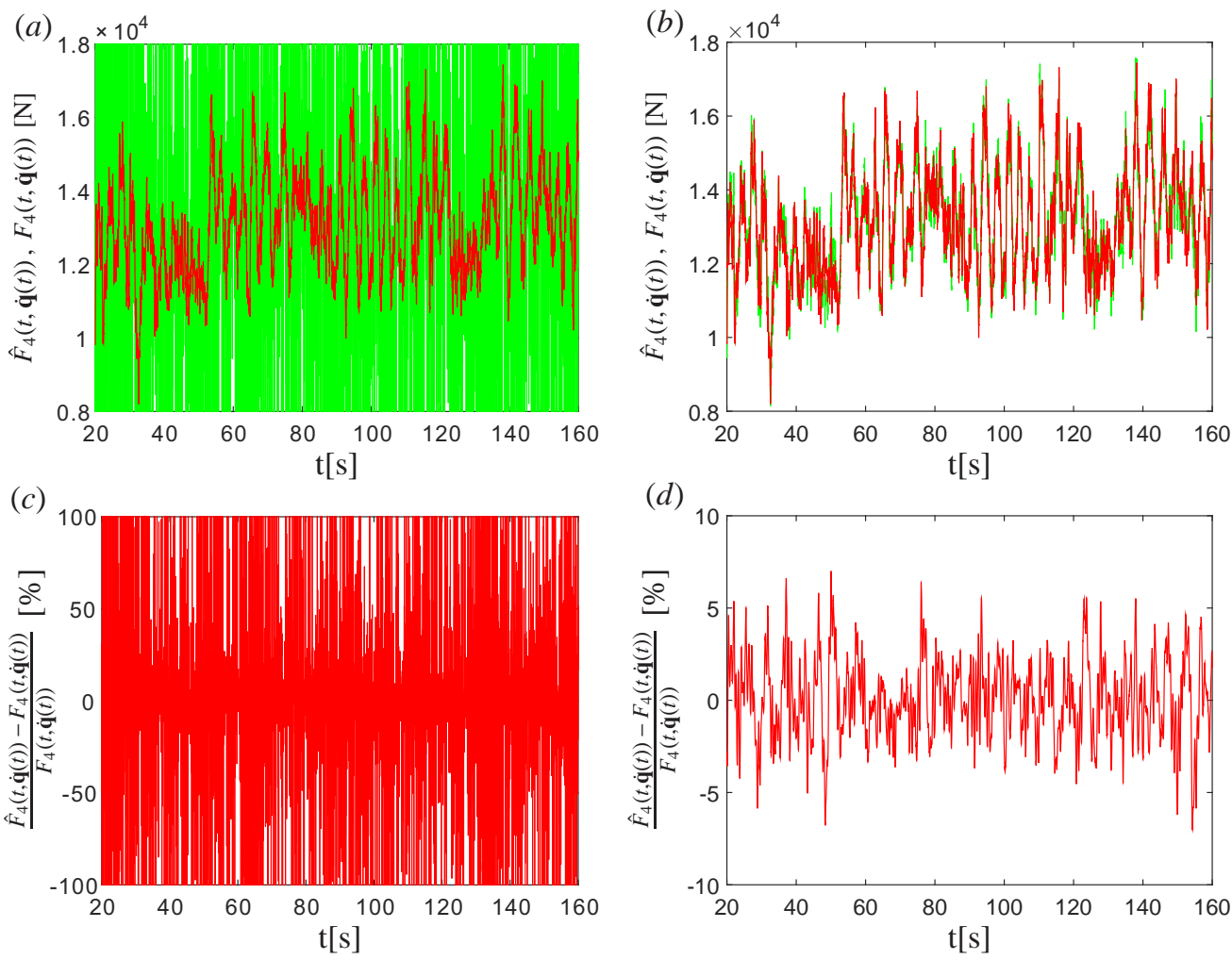

Figure 5: Edgewise load components $\hat{F}_{4}(t, \dot{\mathbf{q}}(t))$ (green), $F_{4}(t, \dot{\mathbf{q}}(t))$ (red) with gravity removed and load relative difference $\frac{\hat{F}_{4}(t, \dot{\mathbf{q}}(t))-F_{4}(t, \dot{\mathbf{q}}(t))}{F_{4}(t, \dot{\mathbf{q}}(t))}$ (red). (a)(c) Wavelet band value $p=5$, (b)(d) Wavelet band value $p=20 . \xi=0$. 
Fig. 4 and Fig. 5 show the comparison between components of the non-linear load $\mathbf{F}(t, \dot{\mathbf{q}}(t))$ (red) and the linearized load vector $\hat{\mathbf{F}}(t, \dot{\mathbf{q}}(t))$ (green) given by Eq. (21) as a function of the number of wavelet bands $p$. Both $\mathbf{F}(t, \dot{\mathbf{q}}(t))$ and $\mathbf{F}_{0}(t)$ have been obtained from the 13-DOF wind turbine model. $\xi$ denotes the noise parameter, which is discussed in section 3.4.

Fig. 4(a)(b) shows the results for the load in the flapwise direction and Fig. 4(c)(d) show the load relative differences. It can be seen from the Fig. 4(a)(c) that for $p=5$, the linearized load $\hat{F}_{1}(t, \dot{\mathbf{q}}(t))$ is quite different from the non-linear load $F_{1}(t, \dot{\mathbf{q}}(t))$. In contrast, for a larger number of wavelet bands $p=20$ the linearized force $\hat{F}_{1}(t, \dot{\mathbf{q}}(t))$ can match well with the non-linear force $F_{1}(t, \dot{\mathbf{q}}(t))$ in Fig. 4(b)(d). This is so because a low $p$ value merely takes the low frequency ingredients of the load into consideration, so the energy contents of the high frequency part is lost. Since the frequency components of the external load vector $\mathbf{F}(t, \dot{\mathbf{q}}(t))$ is simple, $p=20$ should be far more sufficient to meets the accuracy requirements of the $5 \mathrm{MW}$ wind turbine, which is the most widely used structure in current commercial wind turbine industry.

The edgewise external load is dominated by the time-varying gravity contribution. In order to put emphasis on the influence of damping, the gravity force has been skipped in Fig. 5. Actually the same quasi-static gravity force is present in $\hat{F}_{4}(t, \dot{\mathbf{q}}(t))$ and $F_{0,4}(t)$, and will not be present in $\mathbf{C}_{\mathrm{a}}(t) \dot{\mathbf{q}}(t)$. Again, it is seen that the linearized force $\hat{F}_{4}(t, \dot{\mathbf{q}}(t))$ matches the nonlinear force well if a sufficient number $p$ is used. Although not shown here, the same turns out to be true for all other components in $\mathbf{F}(t, \dot{\mathbf{q}}(t))$.

Furthermore, results from Fig. 4(b)(d) and Fig. 5(b)(d) show that the linearized load vector $\hat{\mathbf{F}}(t, \dot{\mathbf{q}}(t))$ computed using wavelet method matches well with the external generalized load vector $\mathbf{F}(t, \dot{\mathbf{q}}(t))$, which implies that there is little differences between the real aerodynamic damping matrix and the identified aerodynamic damping matrix $\mathbf{C}_{\mathrm{a}}(t)$.

\subsection{Estimation of aerodynamic damping using actual measurements}

Above, it has been shown that $\mathbf{C}_{a}(t) \dot{\mathbf{q}}(t)$ can be obtained from the linearization expression in Eq. (8). Hence, $\mathbf{C}_{a}(t) \dot{\mathbf{q}}(t)$ may be estimated provided that $\mathbf{F}(t, \dot{\mathbf{q}}(t))$ and $\mathbf{F}_{0}(t)$ are known. This section proposes an approach for estimating these two load vectors.

It is assumed that the generalised displacement vector $\mathbf{q}(t)$, the generalised velocity vector $\dot{\mathbf{q}}(t)$ and the generalised acceleration vector $\ddot{\mathbf{q}}(t)$ related to a given aeroelastic model can be estimated. Typically, $\ddot{\mathbf{q}}(t)$ is estimated based on acceleration measurements on the structure, and $\mathbf{q}(t)$ and $\dot{\mathbf{q}}(t)$ are obtained by numerical time integration. It is also assumed that the mass matrix $\mathbf{M}(t)$, structural damping matrix $\mathbf{C}(t)$ and stiffness matrix $\mathbf{K}(t)$ are available from the aeroelastic model.

Here we make an assumption that the mass matrix $\mathbf{M}(t)$, structural damping matrix $\mathbf{C}(t)$ and stiffness matrix $\mathbf{K}(t)$ obtained from the aeroelastic model are accurate enough to represent the real wind turbine properties. Actually, unlike other structures (e.g. bridges, buildings or towers), which contains more uncertainties due to their construction methods and materials, the mass and stiffness distribution of wind turbines are much more precisely available, since all parts of the wind turbine are prefabricated in the factory and assembled directly on site. The matrices $\mathbf{M}(t)$ and $\mathbf{K}(t)$ of the wind turbine can be obtained based on specifictions of the wind turbines (e.g. 
for the 5MW turbine NREL Jonkman et al. [24] provides detail information or $10 \mathrm{MW}$ wind turbine information is available from DTU [35]). Such specifications are also available through system identification tests carried out in manufacturing plants (e.g. Bulder et al. [36], Larsen et al. [37], Adams et al. [38], Ghoshal et al. [39], Sundaresan et al. [40], White et al.[41] and Simmermacher et al. [42]). Hence, the $\mathbf{M}(t)$ and $\mathbf{K}(t)$ obtained from the aeroelastic model can be verified by directly comparing with the real $\mathbf{M}(t)$ and $\mathbf{K}(t)$ of the wind turbines. Furthermore, the structural damping matrix $\mathbf{C}(t)$ is numerically much smaller than the aerodyanic damping matrix $\mathbf{C}_{a}(t)$ and has little influence on the overall damping.

Since $\mathbf{M}(t), \mathbf{C}(t), \mathbf{K}(t), \mathbf{q}(t), \dot{\mathbf{q}}(t)$ and $\ddot{\mathbf{q}}(t)$ are available, $\mathbf{F}(t, \dot{\mathbf{q}}(t))$ can be calculated from Eq. (2). $\quad \mathbf{F}_{0}(t)$ is caused by low frequency turbulence and gravity loads, and hence primarily causes a quasi-static response of the structure. Consequently, it may be anticipated that $\mathbf{F}_{s}(t, \mathbf{q}(t))=\mathbf{K}(t) \mathbf{q}(t)$ contains the basic information of $\mathbf{F}_{0}(t)$.

This turns out to the case at most frequencies in the Fourier transform of the components of $\mathbf{F}_{s}(t, \mathbf{q}(t))$, save at the vicinity of the angular eigenfrequency where a dynamic response component may be present. This is especially the case for the components in the load vector conjugated to the edgewise blade modes and the lateral lower mode due to the low aerodynamic damping in these modes. For this reason these dynamic components are filtered out of $\mathbf{F}_{s}(t, \mathbf{q}(t))$ and the resulting load vector is denoted $\mathbf{F}_{f}(t, \mathbf{q}(t))$. For example, it can be seen in Fig. 6(d), there are two resonant areas, which mainly consist of the first and second eigenfrequences of the tower, in $F_{s, 8}(\omega)$. It is observed that the first resonant area starts from $1.4 \mathrm{rad} / \mathrm{s}$ to $2.3 \mathrm{rad} / \mathrm{s}$ and the second area starts from $19.5 \mathrm{rad} / \mathrm{s}$ to $23 \mathrm{rad} / \mathrm{s}$.

The generalised load in the edgewise blade direction $F_{s, 4}\left(t, \mathbf{q}(t), F_{f, 4}\left(t, \mathbf{q}(t)\right.\right.$ and $F_{0,4}(t)$ are dominated by the same quasi-static varying component due to gravity, which is making the load contributions from aerodynamic damping and turbulence much small. For this reason, the gravity has been skipped in the following figures.

Fig. 6(a) shows the Fast Fourier Transformation (FFT) $F_{s, 1}(\omega)$ and $F_{0,1}(\omega)$ of the flapwise load components $F_{s, 1}(t, \mathbf{q}(t))$ and $F_{0,1}(t)$, respectively. Due to the large aerodynamic damping in flapwise direction, influence of the resonant vibrations of the blade in the flapwise direction is negligible. Fig. 6(b) shows the corresponding Fourier transformation of the edgewise load components $F_{s, 4}(t, \mathbf{q}(t))$ and $F_{0,4}(t), F_{s, 4}(\omega)$ is mainly influenced by two resonant components: the first order resonant component of tower and the first order resonant component of blade in edgewise direction in comparison with $F_{0,4}(\omega)$. Unlike $F_{s, 1}(\omega)$, the contribution made by the first order resonant component of blade is much greater than that of the first order resonant component of tower. This is so because the aerodynamic damping of edgewise vibration is much smaller than for flapwise vibration. Fig. 6(c) shows Fourier transformation of the load on the tower in the mean wind direction $F_{s, 7}(t, \mathbf{q}(t))$ and $F_{0,7}(t)$. The resonant component at first order tower eigenfrequency is clearly visible in $F_{s, 7}(\omega)$ despite the relatively large aerodynamic damping of the tower in the along-wind direction. Finally, Fig. 6(d) shows the Fourier transformation of tower load $F_{s, 8}(t, \mathbf{q}(t))$ and $F_{0,8}(t)$ in the lateral direction. Clearly, comparing to $F_{0,8}(t)$, two resonant components of tower make great contribution to $F_{s, 8}(t, \mathbf{q}(t))$.

Fig. 6 shows that the resonant components have significant influence on $\mathbf{F}_{s}(t, \mathbf{q}(t))$, which make the amplitude of $\mathbf{F}_{s}(t, \mathbf{q}(t))$ different from $\mathbf{F}_{0}(t)$ at resonant frequencies. For this reason, 
the Fourier transformation of $\mathbf{F}_{s}(t, \mathbf{q}(t))$ in the vicinity of the eigenfrequency of the tower and the blade, marked with a bold line in Fig. 6, has been filtered out. The remaining part of the Fourier transformation denotes a filtered presentation $\mathbf{F}_{f}(t, \mathbf{q}(t))$ of $\mathbf{F}_{s}(t, \mathbf{q}(t))$, which has a close resemblance to $\mathbf{F}_{0}(t)$.

Fig. 7 shows the comparison between $\mathbf{F}_{f}(t, \mathbf{q}(t))$ (green) and $\mathbf{F}_{0}(t)$ (red). Fig. 8 shows the corresponding relative differences of $\mathbf{F}_{f}(t, \mathbf{q}(t))$ and $\mathbf{F}_{0}(t)$. As seen, $\mathbf{F}_{f}(t, \mathbf{q}(t))$ matches well with $\mathbf{F}_{0}(t)$, not only for the load components in the flapwise and edgewise direction of the blade, but also for the load components in the mean wind direction and lateral direction of tower. Since the values of $F_{f, 8}(t, \mathbf{q}(t))$ and $F_{0,8}(t)$ are fluctuating around 0, Fig. 8(d) shows the value of $\frac{F_{f, 8}(t, \mathbf{q}(t))-F_{0,8}(t)}{\sigma_{F_{0,8}(t)}}$ instead of $\frac{F_{f, 8}(t, \mathbf{q}(t))-F_{0,8}(t)}{F_{0,8}(t)} . \sigma_{F_{0,8}}$ denotes the standard deviation of $F_{0,8}(t)$.

Fig. 9 shows the Fast Fourier transformation of load components of $\mathbf{F}_{f}(t, \mathbf{q}(t))$ and $\mathbf{F}_{0}(t)$. As seen, the frequency ingredients of $\mathbf{F}_{f}(t, \mathbf{q}(t))$ and $\mathbf{F}_{0}(t)$ are almost the same.

Hence, it is concluded that the quasi-static load $\mathbf{F}_{0}(t)$ can be replaced by $\mathbf{F}_{f}(t, \mathbf{q}(t))$ for the estimation of the aerodynamic damping matrix in section 3.1. 

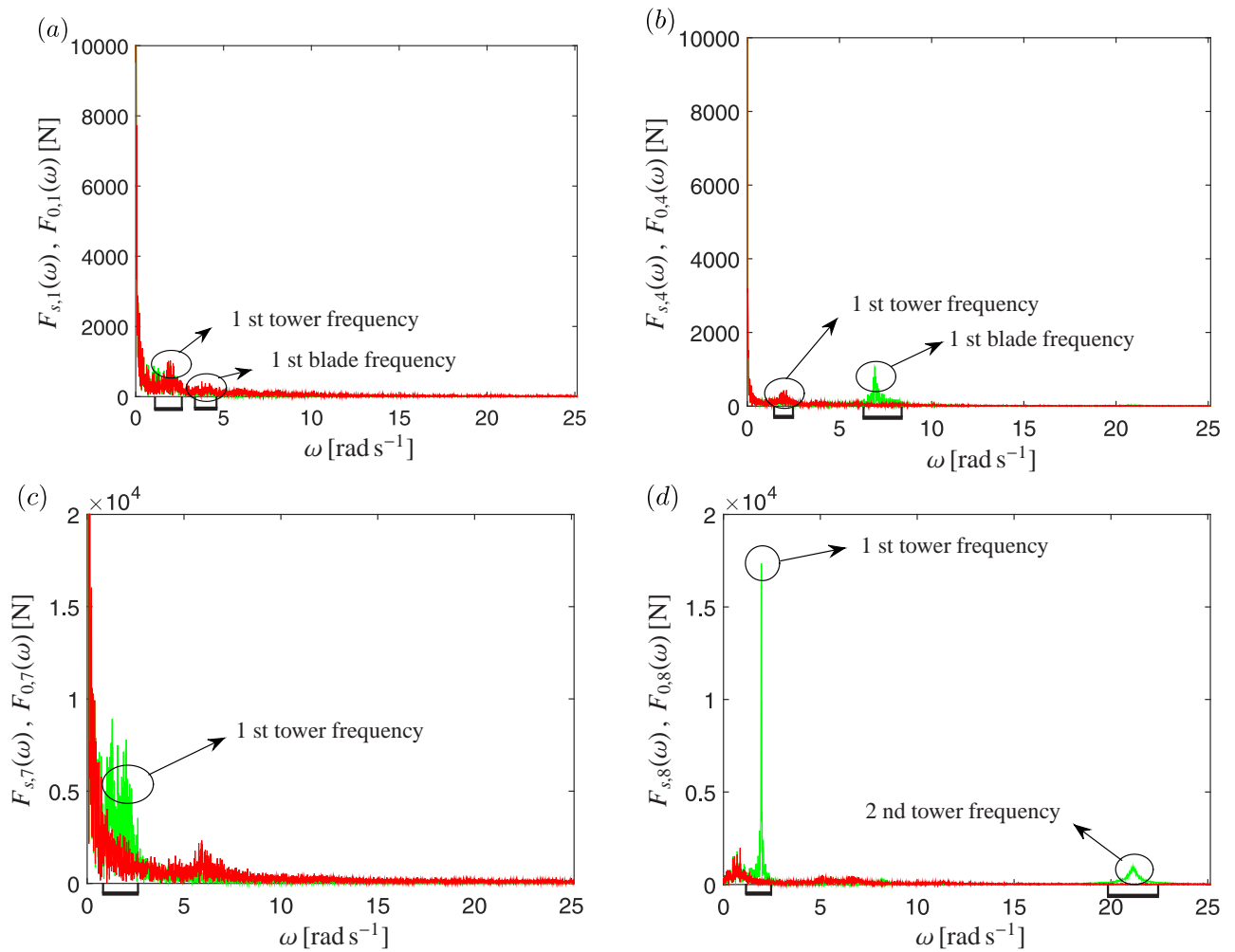

Figure 6: Fourier transformation of load components of $\mathbf{F}_{s}(t, \mathbf{q}(t))$ (green) and $\mathbf{F}_{0}(t)$ (red). (a) Flapwise load components $F_{s, 1}(\omega)$ and $F_{0,1}(\omega)$, (b) Edgewise load components $F_{s, 4}(\omega)$ and $F_{0,4}(\omega)$, (c) Mean wind direction load components on tower $F_{s, 7}(\omega)$ and $F_{0,7}(\omega)$, (d) Lateral load components on tower $F_{s, 8}(\omega)$ and $F_{0,8}(\omega) . \xi=0$. 

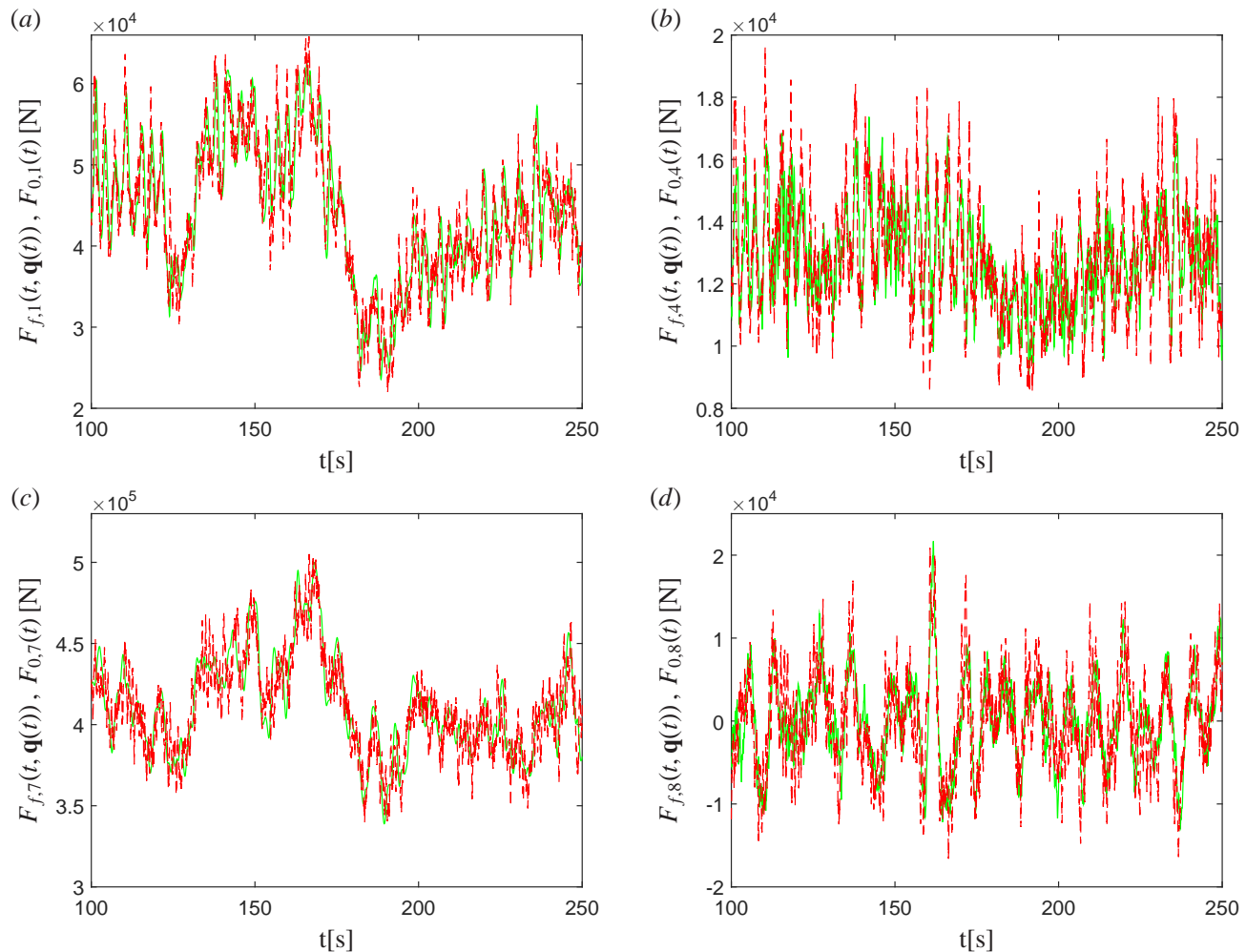

Figure 7: Load components of $\mathbf{F}_{f}(t, \mathbf{q}(t))$ (green) and $\mathbf{F}_{0}(t)$ (red). (a) Flapwise load components $F_{f, 1}(t, \mathbf{q}(t))$ and $F_{0,1}(t)$, (b) Edgewise load components $F_{f, 4}(t, \mathbf{q}(t))$ and $F_{0,4}(t)$, (c) Load components in the mean wind direction on tower $F_{f, 7}(t, \mathbf{q}(t))$ and $F_{0,7}(t),(\mathrm{d})$ Lateral load components on tower $F_{f, 8}(t, \mathbf{q}(t))$ and $F_{0,8}(t) . \xi=0$. 

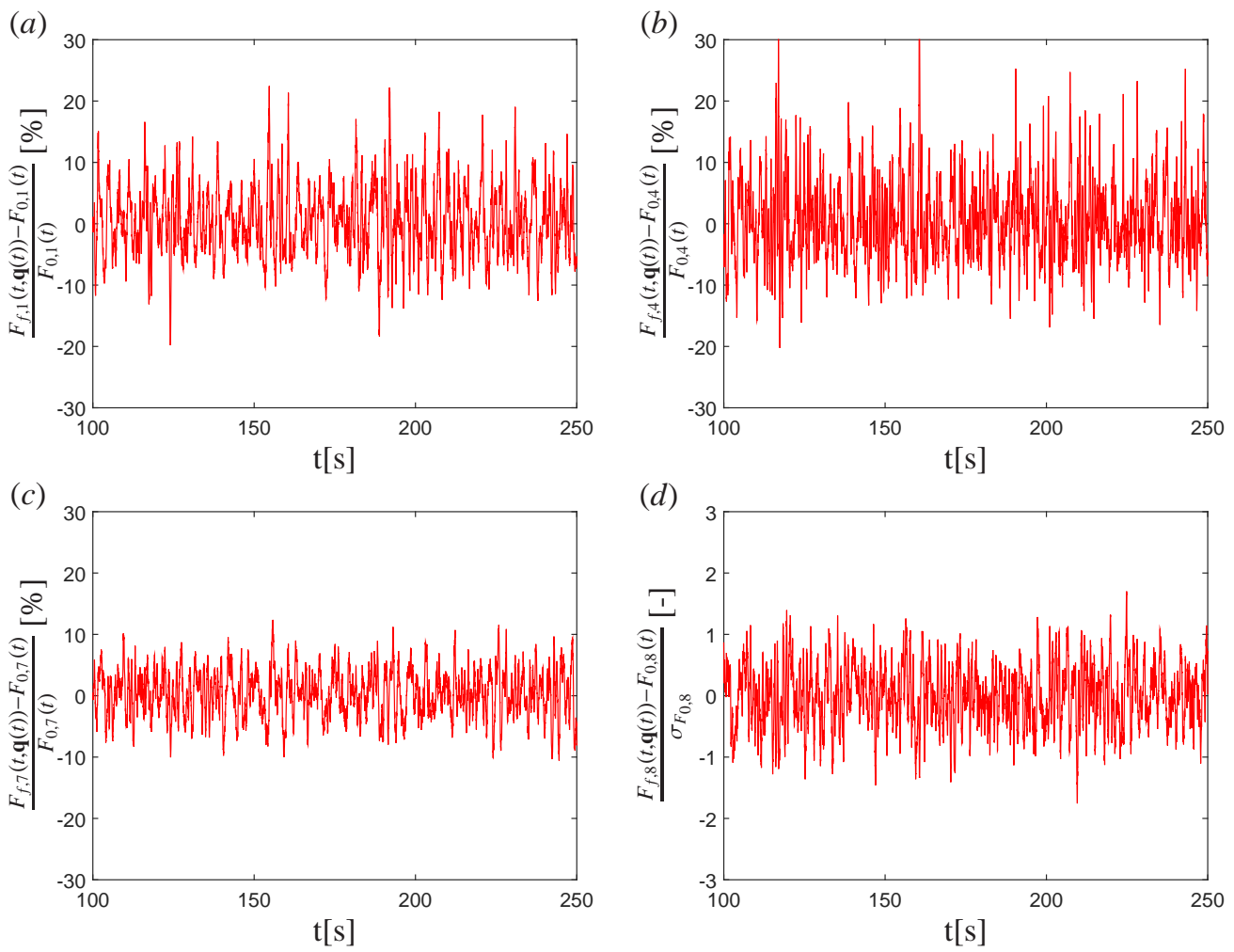

Figure 8: Relative differences of load components. (a)Flapwise load relative difference component $\frac{F_{f, 1}(t, \mathbf{q}(t))-F_{0,1}(t)}{F_{0,1}(t)}$, (b) Edgewise load relative difference component $\frac{F_{f, 4}(t, \mathbf{q}(t))-F_{0,4}(t)}{F_{0,4}(t)}$, (c) Load relative difference component in the mean wind direction on tower $\frac{F_{f, 7}(t, \mathbf{q}(t))-F_{0,7}(t)}{F_{0,7}(t)}$, (d) Lateral Load relative difference component on tower $\frac{F_{f, 8}(t, \mathbf{q}(t))-F_{0,8}(t)}{\sigma_{F_{0,8}}}$. $\xi=0$. 

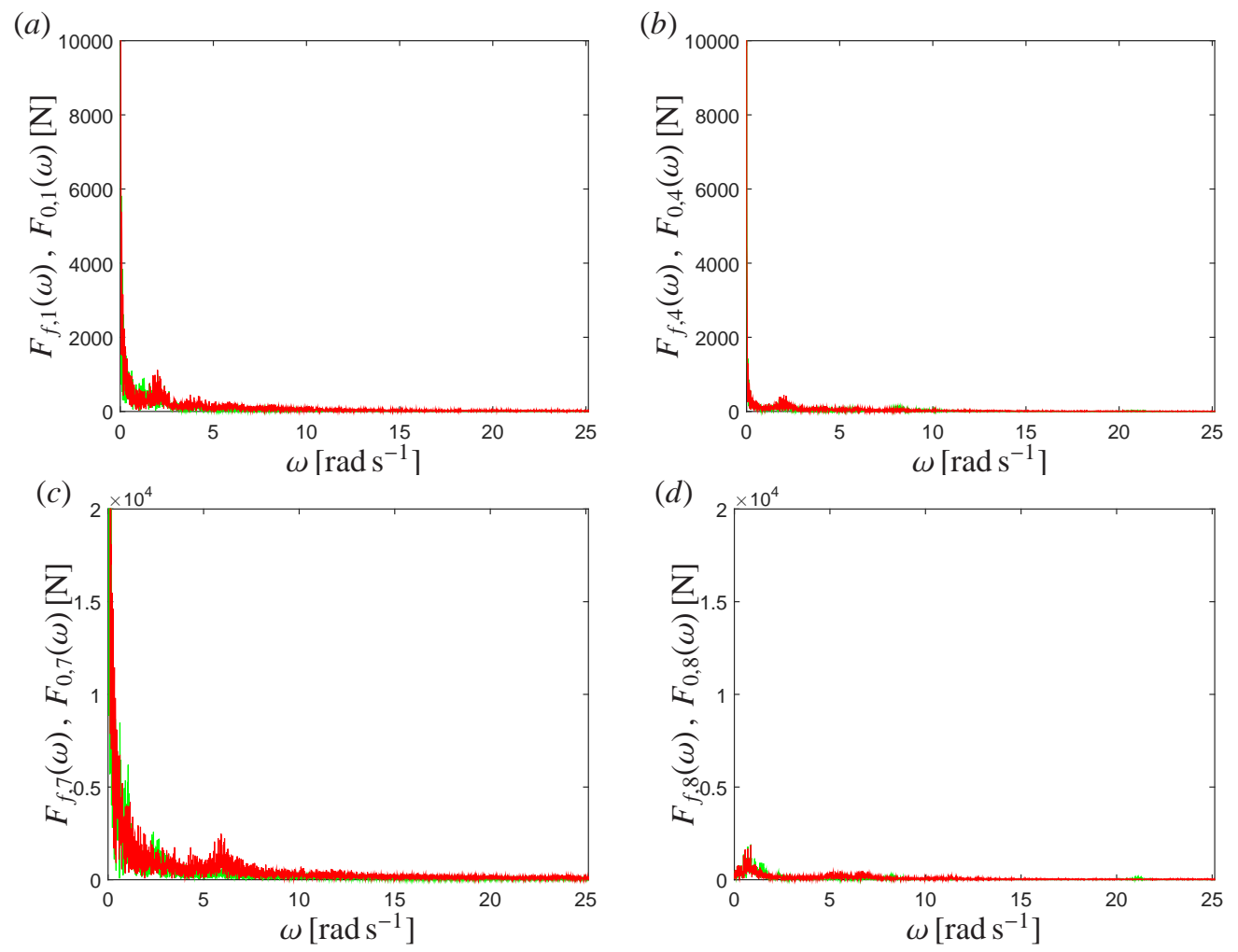

Figure 9: Fourier transformation of load components of $\mathbf{F}_{f}(t, \mathbf{q}(t))$ (green) and $\mathbf{F}_{0}(t)$ (red). (a) Flapwise load components $F_{f, 1}(\omega)$ and $F_{0,1}(\omega)$, (b) Edgewise load components $F_{f, 4}(\omega)$ and $F_{0,4}(\omega)$, (c) Mean wind direction load components on tower $F_{f, 7}(\omega)$ and $F_{0,7}(\omega)$, (d) Lateral load components on tower $F_{f, 8}(\omega)$ and $F_{0,8}(\omega)$. $\xi=0$. 


\subsection{Aerodynamic damping matrix}

Fig. 10 presents the estimation result of the time-varying aerodynamic damping matrixes $\mathbf{C}_{\mathrm{a}, f}(t)$ and $\mathbf{C}_{\mathrm{a}, 0}(t)$. Fig. 11 shows the relative differences between $\mathbf{C}_{\mathrm{a}, f}(t)$ and $\mathbf{C}_{\mathrm{a}, 0}(t)$. $\mathbf{C}_{\mathrm{a}, f}(t)$ is calculated based on the load vector $\mathbf{F}_{f}(t, \mathbf{q}(t))$. $\mathbf{C}_{\mathrm{a}, 0}(t)$, which is calculated based on the load vector $\mathbf{F}_{0}(t)$, is also presented to show the accuracy of the estimation result of the aerodynamic damping matrix $\mathbf{C}_{\mathrm{a}, f}(t)$. For the convenience of representation, only four elements in the main diagonal of $\mathbf{C}_{\mathrm{a}, f}(t)$ and $\mathbf{C}_{\mathrm{a}, 0}(t)$ are plotted as follows.

Fig. 10(a)(b) and Fig. 11(a)(b) show the time history of $C_{\mathrm{a}, f, 1,1}(t)$ (green), $C_{\mathrm{a}, 0,1,1}(t)$ (red), $C_{\mathrm{a}, f, 4,4}(t)$ (green) and $C_{\mathrm{a}, 0,4,4}(t)$ (red) and corresponding relative differences. The solid green line in Fig. 10(a)(b) denotes the mean value, and the dashed green lines denotes the mean value plus or minus one standard deviation of $C_{\mathrm{a}, f, 1,1}(t)$ and $C_{\mathrm{a}, f, 4,4}(t)$, respectively. As seen, $C_{\mathrm{a}, f, 1,1}(t)$ and $C_{\mathrm{a}, f, 4,4}(t)$ are match well with $C_{\mathrm{a}, 0,1,1}(t)$ and $C_{\mathrm{a}, 0,4,4}(t)$, respectively. Furthermore, the value of aerodynamic damping in flapwise direction $C_{\mathrm{a}, f, 1,1}(t)$ is larger than that of edgewise direction $C_{\mathrm{a}, f, 4,4}(t)$, the mean value of $C_{\mathrm{a}, f, 1,1}(t)$ is almost 31 times larger than that of $C_{\mathrm{a}, f, 4,4}(t)$, which is in agreement with the experimental and numerical simulation results in $[1,3]$, and can be explained by the theory based on the quasi-steady aerodynamics [43]. Similar result can also be observed in the following two figures. Fig. $10(\mathrm{c})(\mathrm{d})$ and Fig. 11(c)(d) show the time history of $C_{\mathrm{a}, f, 7,7}(t)$ (green), $C_{\mathrm{a}, 0,7,7}(t)$ (red), $C_{\mathrm{a}, f, 8,8}(t)$ (green) and $C_{\mathrm{a}, 0,8,8}(t)$ (red) and corresponding relative differences, the mean value of aerodynamic damping in mean wind direction $C_{\mathrm{a}, f, 7,7}(t)$ is about $1-2$ times larger than that of $C_{\mathrm{a}, f, 8,8}(t)$ in general. Furthermore, the value of aerodynamic damping of blade is much smaller than that of tower.

As can be seen in these figures, the value of $C_{\mathrm{a}, f, i, i}(t)$ is fluctuating around the mean value dramatically and is not varied periodically, especially for $C_{\mathrm{a}, f, 4,4}(t)$. Furthermore, some estimated values $C_{\mathrm{a}, f, 7,7}(t)$ and $C_{\mathrm{a}, f, 8,8}(t)$ are negative in some time steps. This is so partly because of the time-dependent pitch angle $\beta(t)$, and partly due to the turbulence input as described in section 2.2 . 

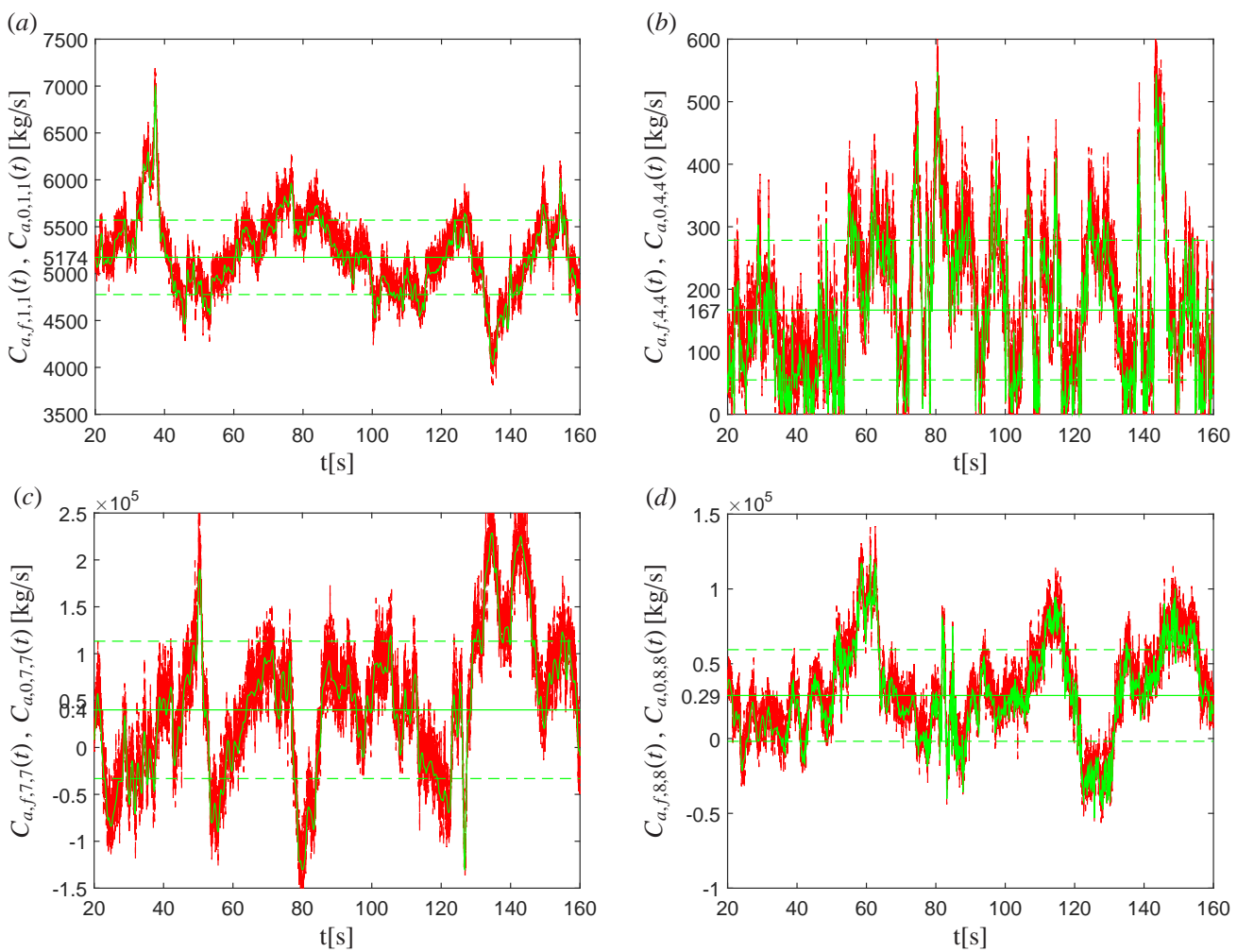

Figure 10: Time series of $\mathbf{C}_{\mathrm{a}, f}(t)$ (green) and mean value of $\mathbf{C}_{\mathrm{a}, f}(t)$ plus or minus one standard deviation of $\mathbf{C}_{\mathrm{a}, f}(t)$. Time series of $\mathbf{C}_{\mathrm{a}, 0}(t)$ (red). (a) $C_{\mathrm{a}, f, 1,1}(t)$ and $C_{\mathrm{a}, 0,1,1}(t)$, (b) $C_{\mathrm{a}, f, 4,4}(t)$ and $C_{\mathrm{a}, 0,4,4}(t)$, (c) $C_{\mathrm{a}, f, 7,7}(t)$ and $C_{\mathrm{a}, 0,7,7}(t),(\mathrm{d}) C_{\mathrm{a}, f, 8,8}(t)$ and $C_{\mathrm{a}, 0,8,8}(t) . \xi=0$. 

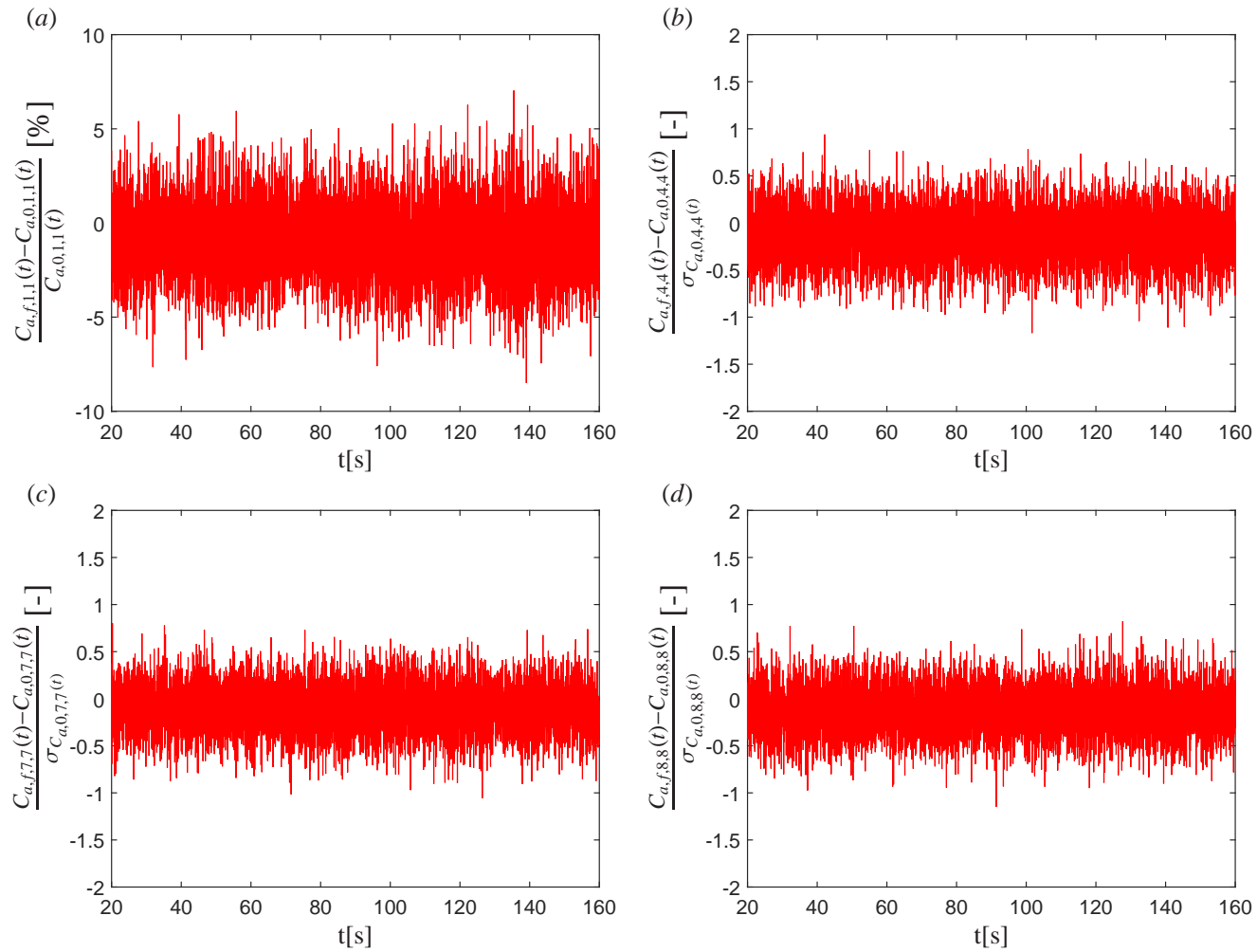

Figure 11: Relative differences of aerodyanmic damping matrix components. (a) $\frac{C_{a, f, 1,1}(t)-C_{a, 0,1,1}(t)}{C_{a, 0,1,1}(t)}$, (b) $\frac{C_{a, f, 4,4}(t)-C_{a, 0,4,4}(t)}{\sigma_{C_{a, 0,4,4}(t)}}$, (c) $\frac{C_{a, f, 7,7}(t)-C_{a, 0,7,7}(t)}{\sigma_{C_{a, 0,7,7}(t)}}$, (d) $\frac{C_{a, f, 8,8}(t)-C_{a, 0,8,8}(t)}{{ } C_{a, 0,8,8}(t)} \cdot \xi=0$. 


\subsection{Effects of noise}

The displacement vector $\mathbf{q}(t)$ and its derivatives $\dot{\mathbf{q}}(t)$ and $\ddot{\mathbf{q}}(t)$ are identified by a linear state observer based on a given number of displacement or acceleration sensor placed on the structure. Hence, the uncertainty related to the estimates of $\mathbf{q}(t), \dot{\mathbf{q}}(t)$ and $\ddot{\mathbf{q}}(t)$ are partly due to estimation errors, and partly due to measurement errors related to the sensor. The noise will be quantified by a non-dimensional parameter $\xi$. Some components of $\mathbf{q}(t)$ have non-zero mean values. In these case $\xi$ indicates the variational component of the signal. For zero-mean components of $\mathbf{q}(t)$ and all the components of $\dot{\mathbf{q}}(t)$ and $\ddot{\mathbf{q}}(t), \xi$ is defined as a fraction of the standard deviation of these components. All non-zero mean values and standard deviation are determined based on ergodic sampling of the time series obtained from the aeroelastic model.

The component noise processes of $\mathbf{q}(t), \dot{\mathbf{q}}(t)$ and $\ddot{\mathbf{q}}(t)$ are modelled as a broad-banded mutual independent zero-mean Gaussian process. The stochastic model of the broad-banded process is chosen as a so-called broken line process with a realization shown on Fig. 12, which mimics Gaussian white noise process in the sense that the auto-spectral density is flat at all angular frequency of structural importance [44]. The variation of the process can be adjusted to provide a given standard derived by calibrating the time step $\Delta t$ and the standard deviation $\sigma_{w}$ of the zeromean mutual independent Gaussian random variables $w_{\mathrm{j}} . \alpha_{\mathrm{b}}$ is a stochastic variable uniformly distributed in the interval $[0,1]$, stochastic independent of all $w_{\mathrm{j}}$. The variance of the broken line process can be shown to be $\frac{2}{3} \sigma_{w}^{2}$ [44]. In this numerical case, we choose $\Delta t=\frac{1}{50} T_{4}$, where $T_{4}=\frac{2 \pi}{\omega_{4}}$ and $\omega_{4}$ denotes the angular edgewise eigenfrequency of the blade, which is the highest angular eigenfrequency of the structure. Fig. 13 shows the comparison between the original signal $q_{1}(t)$ and the signal $q_{1}(t)$ with a noise component superposed.

Since the noise has much high frequency ingredients than the underlying signal, higher value of wavelet bands $p$ is chosen (i.e. in this case $p=21$ is sufficient).

Fig. 14 shows the mean value of four aerodynamic damping matrix elements plus or minus one standard deviation under different noise conditions. Fig. 15 shows the corresponding time series curves. $\xi=0$ denotes the situation where noise is not considered. As seen, the estimation results are not severely sensitive to the noise. 


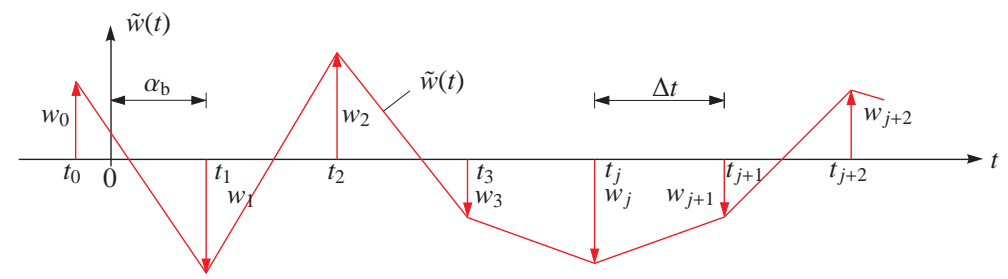

Figure 12: Realization of the broken line process.

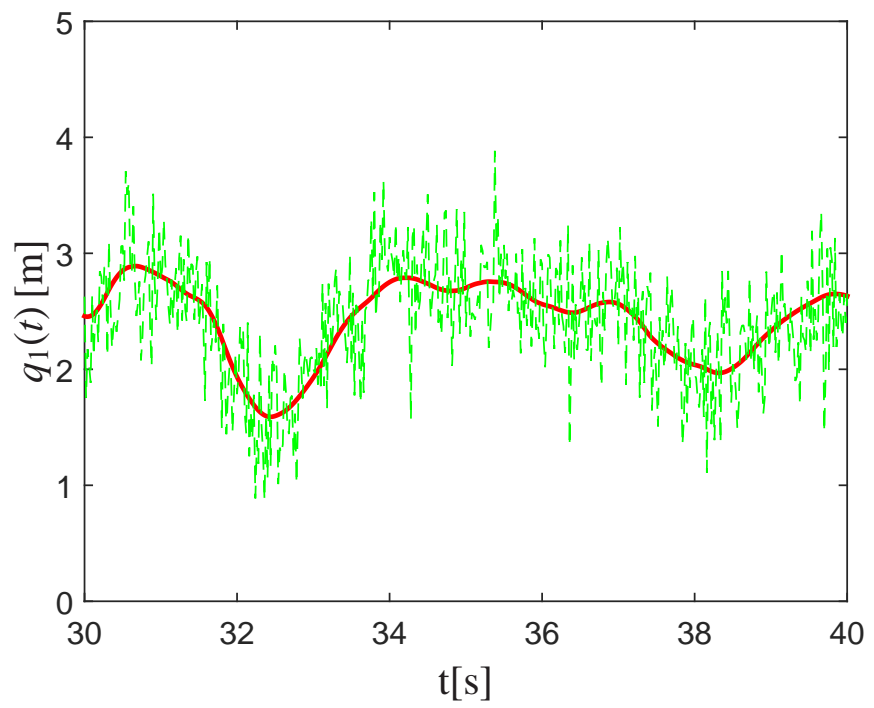

Figure 13: Time series of flapwise tip displacement $q_{1}(t)$. Red curve: $\xi=0$. Green curve: with superposed noise, $\xi=0.5$. 

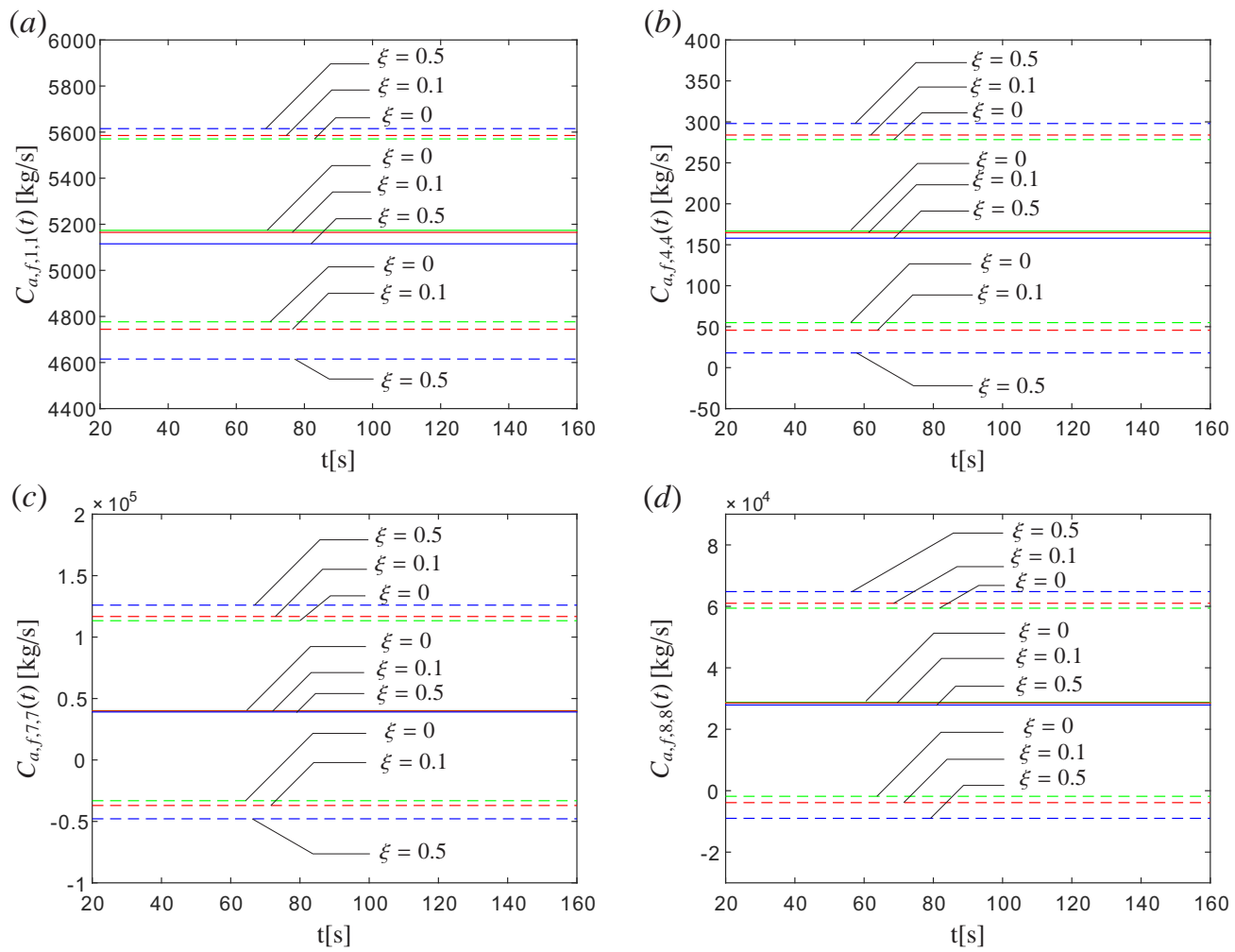

Figure 14: Mean value of $\mathbf{C}_{\mathrm{a}, f}(t)$ plus or minus one standard deviation under different noise conditions. $\xi=0$ (green), $\xi=0.1$ (red), $\xi=0.5$ (blue). (a) $C_{\mathrm{a}, f, 1,1}(t),(\mathrm{b}) C_{\mathrm{a}, f, 4,4}(t)$, (c) $C_{\mathrm{a}, f, 7,7}(t),(\mathrm{d}) C_{\mathrm{a}, f, 8,8}(t)$. 

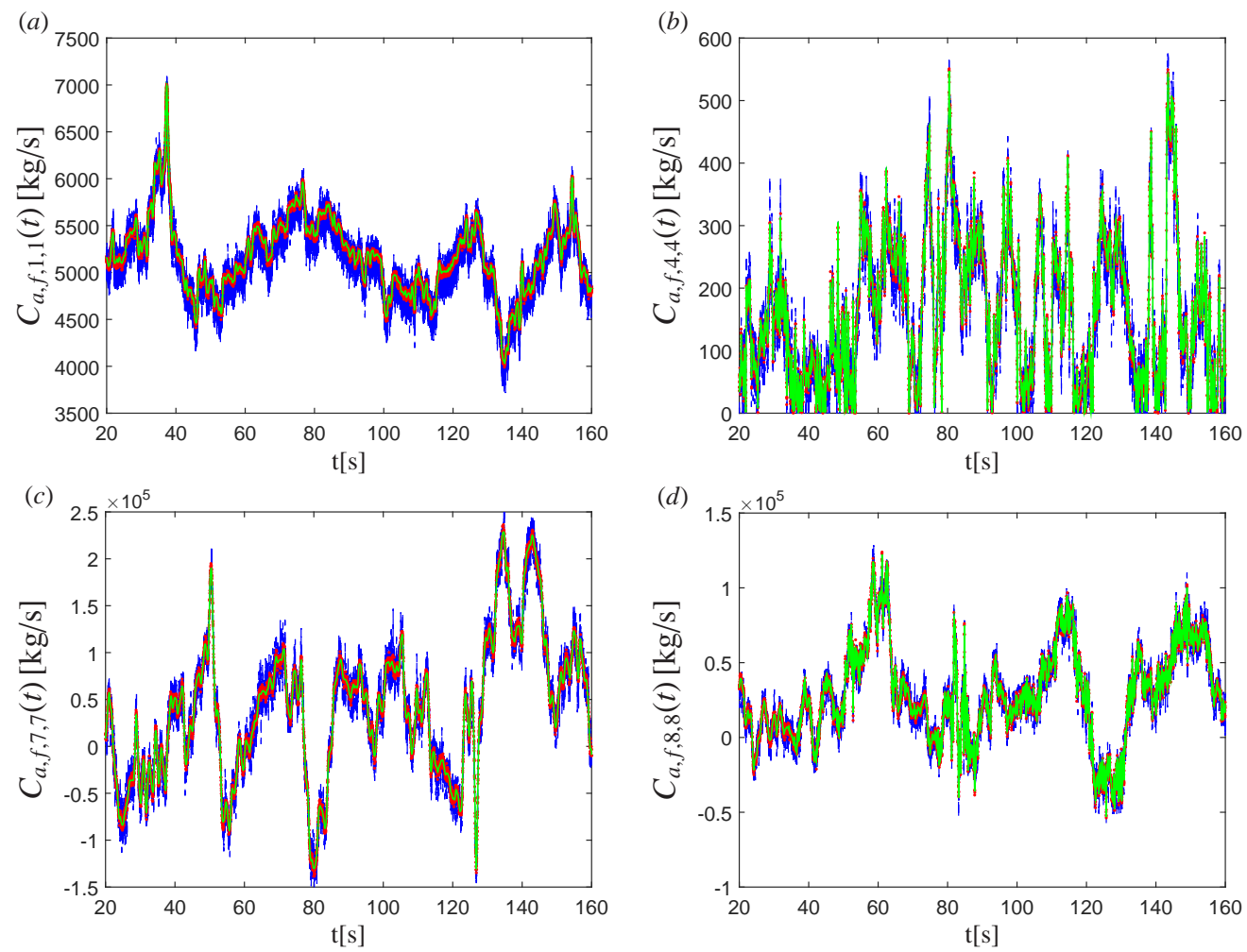

Figure 15: Time series of $\mathbf{C}_{\mathrm{a}, f}(t)$ under different noise conditions. $\xi=0$ (green), $\xi=0.1$ (red), $\xi=0.5$ (blue). (a) $C_{a, f, 1,1}(t),(\mathrm{b}) C_{a, f, 4,4}(t),(\mathrm{c}) C_{a, f, 7,7}(t),(\mathrm{d}) C_{a, f, 8,8}(t)$. 
Data from the NREL 5MW reference wind turbine [24] have been used to calibrate the structural model. The constant parameters employed in the 13-DOF wind turbine model are calculated and provided in Table 1.

Table 1: Parameters in the 13-DOF wind turbine model.

\begin{tabular}{|lll|lll|}
\hline Parameter & Value & Unit & Item & Value & Unit \\
\hline \hline$L_{\mathrm{B}}$ & 61.5 & $\mathrm{~m}$ & 1 st flapwise frequency & 0.669 & $\mathrm{~Hz}$ \\
$s$ & 2.5 & $\mathrm{~m}$ & 1 st edgewise frequency & 1.062 & $\mathrm{~Hz}$ \\
$H_{0}$ & 77.60 & $\mathrm{~m}$ & 1 st tower fore-aft frequency & 0.280 & $\mathrm{~Hz}$ \\
$\Omega$ & 1.27 & $\mathrm{rad} / \mathrm{s}$ & 1 st tower lateral frequency & 0.280 & $\mathrm{~Hz}$ \\
$g$ & 9.81 & $\mathrm{~m} / \mathrm{s}^{2}$ & & & \\
$\rho$ & $1.0 \cdot 10^{3}$ & $\mathrm{~kg} / \mathrm{m}^{3}$ & & & \\
\hline
\end{tabular}




\section{Conclusions}

This paper presents an approach for identification of aerodynamic damping in wind turbines during operation. The proposed method is based on the actual measurements of the response of the wind turbine from which the generalised displacement, velocity and acceleration vectors related to a given aeroelastic model may be estimated by a state observer. The details of the estimation procedure was not reported in the paper. Then, based on the aeroelastic model the external generalised load vector can be obtained from the equation of motion inherent in the aeroelastic code. Additionally, the approach requires the quasi-static load vector, which represents the general load vector, when the elastic deformation of the wind turbine is set to zero. It is demonstrated that the quasi-static load vector can be obtained by filtering out the resonant components in the elastic force vector made up by the product of the stiffness matrix and the generalised displacement vector.

Further, the wavelet-based linearization technique has been used for estimating the aerodynamic damping matrix. The technique breaks down the generalised load vector and the linearized load vector into $2 D$ sequences. From there, the aerodynamic damping matrix is obtained by minimizing the error term between the non-linear generalised load vector and the linearized load vector at each time step, using the least square approach and a smoothing technique. Furthermore, it is demonstrated that the noise has little effect on the estimation results. This technique has the capacity of continuous tracking of the long time variation of the aerodynamic damping matrix, and also avoid calculating the inverse of the singular covariance matrix of velocity vector.

The effectiveness and accuracy of the proposed method has been verified numerically through a reduced 13-DOF aeroelastic model, which is supposed to mimic the real turbine in a turbulent inflow to the rotor.

\section{Appendix A. Definition of aerodynamic damping}

Generally speaking, when a wind turbine blade vibrates in a natural mode, the aerodynamic force on a section of the blade might have a component, which is proportional to the vibrational velocity of the blade section. Here we are interested in force terms arising in connection with velocities superimposed on the dominating velocity associated with rigid body rotation of the blade due to rotor rotation. This principal velocity component together with the free wind velocity gives the main contribution to the force on the blade section. Velocities superimposed on the principal velocity contribute with a force, which with good approximation can be considered proportional to the superimposed velocity, and therefore act as a damping force usually denoted the aerodynamic damping [31].

\section{Acknowledgements}

The first author gratefully acknowledges the financial support from the Chinese Scholarship Council under the State Scholarship Fund. The support of National Science foundation of China is also greatly acknowledged (No. 51278189, No. 51422806). 


\section{References}

[1] M. H. Hansen, Aeroelastic instability problems for wind turbines, Wind Energy 10 (6) (2007) 551-577.

[2] M. O. L. Hansen, J. N. Sørensen, S. Voutsinas, N. Sørensen, H. A. Madsen, State of the art in wind turbine aerodynamics and aeroelasticity, Progress in aerospace sciences 42 (4) (2006) 285-330.

[3] P. Chaviaropoulos, Flap/lead-lag aeroelastic stability of wind turbine blades, Wind Energy 4 (4) (2001) 183-200.

[4] F. Rasmussen, J. T. Petersen, H. A. Madsen, Dynamic stall and aerodynamic damping, Journal of Solar Energy Engineering 121 (3) (1999) 150-155.

[5] M. Hansen, Aeroelastic stability analysis of wind turbines using an eigenvalue approach, Wind Energy 7 (2) (2004) $133-143$.

[6] D. A. Peters, Fast floquet theory and trim for multi-bladed rotorcraft, Journal of the American Helicopter Society 39 (4) (1994) 82-89.

[7] M. J. Kühn, Dynamics and design optimisation of offshore wind energy conversion systems, TU Delft, Delft University of Technology, 2001.

[8] J. Van Der Tempel, Design of support structures for offshore wind turbines, TU Delft, Delft University of Technology, 2006.

[9] D. Salzmann, J. Van der Tempel, Aerodynamic damping in the design of support structures for offshore wind turbines, in: Paper of the Copenhagen offshore conference, 2005.

[10] G. H. James III, T. G. Carne, J. P. Lauffer, The natural excitation technique (NExT) for modal parameter extraction from operating wind turbines, Tech. rep., Sandia National Labs., Albuquerque, NM (United States) (1993).

[11] H. Vold, T. Rocklin, The numerical implementation of a multi-input modal estimation algorithm for minicomputers, Proceedings of the 1st IMAC, Orlando, FL.

[12] M. H. Hansen, K. Thomsen, P. Fuglsang, T. Knudsen, Two methods for estimating aeroelastic damping of operational wind turbine modes from experiments, Wind Energy 9 (1-2) (2006) 179-191.

[13] K. Thomsen, J. T. Petersen, E. Nim, S. Øye, B. Petersen, A method for determination of damping for edgewise blade vibrations, Wind Energy 3 (4) (2000) 233-246.

[14] M. Ruzzene, A. Fasana, L. Garibaldi, B. Piombo, Natural frequencies and dampings identification using wavelet transform: application to real data, Mechanical Systems and Signal Processing 11 (2) (1997) 207-218.

[15] A. Chakraborty, B. Basu, M. Mitra, Identification of modal parameters of a mdof system by modified L-P wavelet packets, Journal of Sound and Vibration 295 (3) (2006) 827-837.

[16] C. H. Lamarque, S. Pernot, A. Cuer, Damping identification in multi-degree-of-freedom systems via a waveletlogarithmic decrementpart 1: theory, Journal of Sound and Vibration 235 (3) (2000) 361-374.

[17] S. Hans, E. Ibraim, S. Pernot, C. Boutin, C.-H. Lamarque, Damping identification in multi-degree-of-freedom system via a wavelet-logarithmic decrementpart 2: study of a civil engineering building, Journal of Sound and Vibration 235 (3) (2000) 375-403.

[18] Q. Li, J. Wu, Time-frequency analysis of typhoon effects on a 79-storey tall building, Journal of Wind Engineering and Industrial Aerodynamics 95 (12) (2007) 1648-1666.

[19] T. H. Le, L. Caracoglia, Reduced-order wavelet-Galerkin solution for the coupled, nonlinear stochastic response of slender buildings in transient winds, Journal of Sound and Vibration 344 (2015) 179-208.

[20] M. Takeuchi, J. Maeda, N. Ishida, Aerodynamic damping properties of two transmission towers estimated by combining several identification methods, Journal of Wind Engineering and Industrial Aerodynamics 98 (12) (2010) $872-880$.

[21] H. Wang, J. Mao, J. Huang, A. Li, Modal identification of sutong cable-stayed bridge during typhoon Haikui using wavelet transform method, Journal of Performance of Constructed Facilities (2016) 04016001.

[22] P. Murtagh, B. Basu, Identification of equivalent modal damping for a wind turbine at standstill using Fourier and wavelet analysis, Proceedings of the Institution of Mechanical Engineers, Part K: Journal of Multi-body Dynamics 221 (4) (2007) 577-589.

[23] Z. Zhang, S. R. K. Nielsen, F. Blaabjerg, D. Zhou, Dynamics and control of lateral tower vibrations in offshore wind turbines by means of active generator torque, Energies 7 (11) (2014) 7746-7772.

[24] J. M. Jonkman, S. Butterfield, W. Musial, G. Scott, Definition of a 5-MW reference wind turbine for offshore system development (2009).

[25] M. O. Hansen, Aerodynamics of wind turbines, Routledge, 2015.

[26] I. E. Commission, et al., Wind turbinepart 1: Design requirements, IEC 61400-1, International Electrotechnical Commission, Geneva, Switzerland.

[27] G. K. Batchelor, The theory of homogeneous turbulence, Cambridge university press, 1953.

[28] S. Krenk, Explicit calibration and simulation of stochastic fields by low-order ARMA processes, in: Eccomas Thematic Conference-Compdyn 2011, 2011.

[29] J. R. Connell, The spectrum of wind speed fluctuations encountered by a rotating blade of a wind energy conversion system, Solar Energy 29 (5) (1982) 363-375. 
[30] M. O. L. Hansen, Aerodynamics of wind turbine, Earthscan, London, UK, 2008.

[31] J. Thirstrup Petersen, H. Aagaard Madsen, A. Björck, P. Enevoldsen, S. Øye, H. Ganander, D. Winkelaar, Prediction of dynamic loads and induced vibrations in stall, Tech. rep. (1998).

[32] C. K. Chui, An introduction to wavelets, Vol. 1, Academic press, 2014.

[33] B. Basu, V. Gupta, On equivalent linearization using wavelet transform, Journal of vibration and acoustics 121 (4) (1999) 429-432.

[34] B. Basu, V. K. Gupta, Seismic response of SDOF systems by wavelet modeling of nonstationary processes, Journal of Engineering Mechanics 124 (10) (1998) 1142-1150.

[35] C. Bak, F. Zahle, R. Bitsche, T. Kim, A. Yde, L. C. Henriksen, A. Natarajan, M. Hansen, Description of the DTU 10 MW reference wind turbine, DTU Wind Energy Report-I-0092 5.

[36] B. Bulder, J. van Dam, D. van Delft, E. Jørgensen, V. Kolovos, S. Larwood, W. Musial, A. Verheul, P. Vionis, M. Winther-Jensen, European wind turbine testing procedure development blade test methods and techniques, Wind Energy for the Next Millennium. Proceedings.

[37] G. C. Larsen, M. H. Hansen, A. Baumgart, I. Carlén, Modal analysis of wind turbine blades, Tech. rep. (2002).

[38] D. Adams, J. White, M. Rumsey, C. Farrar, Structural health monitoring of wind turbines: method and application to a HAWT, Wind Energy 14 (4) (2011) 603-623.

[39] A. Ghoshal, M. J. Sundaresan, M. J. Schulz, P. F. Pai, Structural health monitoring techniques for wind turbine blades, Journal of Wind Engineering and Industrial Aerodynamics 85 (3) (2000) 309-324.

[40] M. Sundaresan, M. Schulz, A. Ghoshal, Structural health monitoring static test of a wind turbine blade subcontract report NREL, Tech. rep., SR-500-28719. Colorado (2002).

[41] J. R. White, D. E. Adams, M. A. Rumsey, Modal analysis of CX-100 rotor blade and micon 65/13 wind turbine, in: Structural Dynamics and Renewable Energy, Volume 1, Springer, 2011, pp. 15-27.

[42] T. Simmermacher, T. Carne, Tower modal survey, Internal SNL Memo, Sandia National Labs, Albuquerque, NM, Dec 20.

[43] M. Hansen, Improved modal dynamics of wind turbines to avoid stall-induced vibrations, Wind Energy 6 (2) (2003) 179-195.

[44] R. W. Clough, J. Penzien, Dynamics of structures. 1993, Copyright of Applied Mechanics \& Materials. 